\title{
Flow and Mixing Fields for Transported Scalar PDF Simulations of a Piloted Jet Diffusion Flame ('Delft Flame III')
}

\author{
BART MERCI ${ }^{1,2}$, BERTRAND NAUD $^{3,4}$ and DIRK ROEKAERTS ${ }^{5,6}$ \\ ${ }^{1}$ Ghent University, Department of Flow, Heat and Combustion Mechanics, Ghent (Belgium), \\ Sint-Pietersnieuwstraat 41, B-9000 Ghent,Belgium; E-mail: bart.merci@UGent.be \\ ${ }^{2}$ Postdoctoral Researcher of the Fund of Scientific Research - Flanders, Belgium (FWO-Vlaanderen) \\ ${ }^{3}$ LITEC-CSIC, Zaragoza, Spain \\ ${ }^{4}$ CIEMAT, Madrid, Spain \\ ${ }^{5}$ Delft University of Technology, Department of Multi Scale Physics, Delft, The Netherlands \\ ${ }^{6}$ Shell Global Solutions International BV, Amsterdam, The Netherlands
}

Received 23 August 2004; accepted 14 December 2004

\begin{abstract}
Numerical simulation results are presented for 'Delft Flame III', a piloted jet diffusion flame with strong turbulence-chemistry interaction. While pilot flames emerge from 12 separate holes in the experiments, the simulations are performed on a rectangular grid, under the assumption of axisymmetry. In the first part of the paper, flow and mixing field results are presented with a non-linear first order $k-\varepsilon$ model, with the transport equation for $\varepsilon$ based on a modeled enstrophy transport equation, for cold and reactive flows. For the latter, the turbulence model is applied in combination with pre-assumed $\beta$-PDF modeling for the turbulence-chemistry interaction. The mixture fraction serves as conserved scalar. Two chemistry models are considered: chemical equilibrium and a steady laminar flamelet model. The importance of the turbulence model is highlighted. The influence of the chemistry model is noticeable too. A procedure is described to construct appropriate inlet boundary conditions. Still, the generation of accurate inlet boundary conditions is shown to be far less important, their effect being local, close to the nozzle exit. In the second part of the paper, results are presented with the transported scalar PDF approach as turbulencechemistry interaction model. A $\mathrm{C}_{1}$ skeletal scheme serves as chemistry model, while the EMST method is applied as micro-mixing model. For the transported PDF simulations, the model for the pilot flames, as an energy source term in the mean enthalpy transport equation, is important with respect to the accuracy of the flow field predictions. It is explained that the strong influence on the flow and mixing field is through the turbulent shear stress force in the region, close to the nozzle exit.
\end{abstract}

Key words: transported PDF, non-linear k-epsilon model, Delft Fame III, turbulent non-premixed combustion

\section{Nomenclature}

$A=$ Cell surface area $\left(\mathrm{m}^{2}\right)$

$f=$ Probability density function (-)

$F=$ Mass density function $\left(\mathrm{kg} / \mathrm{m}^{3}\right)$ 
$g=$ Mixture fraction variance $(-)$

$h=$ Static enthalpy $(\mathrm{J} / \mathrm{kg})$

$k=$ Turbulent kinetic energy $\left(\mathrm{m}^{2} / \mathrm{s}^{2}\right)$

$P_{\mathrm{k}}=$ Production rate of turbulent kinetic energy $\left(\mathrm{m}^{2} / \mathrm{s}^{3}\right)$

$Q=$ Volumetric power $\left(\mathrm{W} / \mathrm{m}^{3}\right)$

$S_{\mathrm{ij}}=i j$-Component of strain rate tensor $(1 / \mathrm{s})$

$S=$ Strain rate $(1 / \mathrm{s})$

$t=$ Time (s)

$T=$ Temperature $(\mathrm{K})$

$U=$ Velocity $(\mathrm{m} / \mathrm{s})$

$v_{\mathrm{i}}=i$-Component of velocity vector $(\mathrm{m} / \mathrm{s})$

$W=$ Molecular weight $(\mathrm{kg} / \mathrm{mol})$

$x_{\mathrm{i}}=($ Eulerian $) i$-coordinate $(\mathrm{m})$

$\mathrm{X}_{\mathrm{i}}=$ (Lagrangian) position vector $(\mathrm{m})$

$Y=$ Species mass fraction $(-)$

$\chi=$ Scalar dissipation rate $(1 / \mathrm{s})$

$\Gamma_{\mathrm{t}}=$ Turbulent diffusivity (Pa.s)

$\delta_{\mathrm{ij}}=$ Kronecker delta (-)

$\varepsilon=$ Turbulent dissipation rate $\left(\mathrm{m}^{2} / \mathrm{s}^{3}\right)$

$\mu=$ Viscosity (Pa.s)

$\rho=$ Density $\left(\mathrm{kg} / \mathrm{m}^{3}\right)$

$\sigma=$ Prandtl number/Schmidt number (-)

$\tau_{\mathrm{t}}=$ Turbulent time scale (s)

$\xi=$ Mixture fraction (-)

$\zeta=$ Standardized Gaussian random variable $(-)$

\section{Introduction}

In this paper, numerical results are presented for the piloted jet diffusion flame, known as 'Delft Flame III' [1-4]. This flame, with relatively strong interaction between turbulence and chemistry, is a target test flame in the series 'International Workshop on Measurements and Computations of Turbulent Non-premixed Flames (TNF)' [5]. The most detailed modelling study published so far is [2]. In an early stage, when experimental work was still ongoing, Delft Flame III has been studied at the 2nd ASCF workshop (Aerodynamics of Steady State Combustion Chambers), organised by a special interest group of ERCOFTAC in Pisa, Italy in 1996. Simulation results are presented in the final report of the workshop [6] and one related conference paper [7]. Results of the combination of $k-\varepsilon$ model with round jet correction and presumed shape mixture fraction PDF models are contained in [1] and [8]. However, the experimental data of mean temperature used for validation purposes in these early works in the mean time have been superseded by more accurate measurements $[3,4]$. As a consequence, when comparing with the new measurements the agreement with the assumed shape PDF results is not so good as 
found in $[1,7,8]$. In this work the performance of presumed PDF models will be reconsidered.

In the first part of this paper, the relative importance of the turbulence model choice, the chemistry model and the construction of accurate inlet boundary conditions on the global quality of the simulation results is discussed in the framework of pre-assumed $\beta$-PDF ('Probability Density Function') modeling for turbulencechemistry interaction, with the mixture fraction as conserved scalar. First, a nonlinear $k-\varepsilon$ turbulence model, that has been applied to a variety of flow types without test case dependent model parameter tuning [9-12], is used. Originally, this model has been developed up to third order, but further experience has revealed that only the first order part needs to be retained [13]. For flows with rotation effects or strong streamline curvature, it is important that the definition of the eddy viscosity contains a non-linear dependence on local strain rate and vorticity, but for the test case under study this is not relevant. The important model feature is the transport equation for the dissipation rate $\varepsilon$ : following the idea of Shih et al. [14], this transport equation is based on a modeled transport equation for mean enstrophy (i.e. mean of the square of the vorticity fluctuations). The sensitivity to inlet boundary conditions and stability problems near solid boundaries are avoided in the model formulation of [11]. The turbulence model is very briefly described in Section 3.1 and in the appendix.

Next, the impact of the chemistry model is considered through a comparison of results obtained with a steady laminar flamelet model to the chemical equilibrium model results.

Furthermore, the influence of the inlet boundary conditions is investigated. On the one hand, separate calculations are performed on fine grids, inside the nozzle. The profiles obtained at the nozzle exit are then used as inlet profiles for the actual flame calculations. The procedure to construct the inlet profiles is described in Section 4.2. Results with these inlet boundary conditions are compared to results when the profiles, used in [2], are applied. This comparison reveals that the influence of the inlet boundary conditions is certainly visible, close to the burner head, but it is far less important than the choice of the turbulence model.

As mentioned, all the above is performed with pre-assumed $\beta$-PDF ('PPDF') modeling of the turbulence-chemistry interaction. In the second part of the paper, transported PDF ('TPDF') [15] results are discussed. Only scalar quantities are used as independent density variables ('hybrid approach'). The same turbulence model as for the PPDF calculations is applied. As chemistry model, the $\mathrm{C}_{1}$ skeletal scheme of [16] is used. The EMST model [17] is applied to model the micro-mixing term. Under these circumstances, the flame does not ignite automatically, in contrast to the PPDF simulations with chemical equilibrium. The pilot flames are modeled as a volumetric energy source term in the mean enthalpy transport equation, as described in Section 3.4. It is illustrated that the position of the region where this source term is added, strongly affects the quality of the flow and mixing field predictions near the burner head, due to qualitatively different fields of turbulent kinetic energy and 
turbulent shear stress. Further downstream, this influence becomes small, as long as the flame ignites.

Finally, we remark that the focus of the second part of this paper is on the influence of the choice of the pilot flame model, turbulence model and inlet boundary conditions. In [18], the detailed quality of the transported PDF results with respect to the micro-mixing term is discussed and the choices of flow and pilot flames model and inlet boundary conditions are justified by the results and discussion of the current paper.

\section{Test Case Description}

Figure 1 shows the burner head of Delft Flame III. The fuel, Dutch natural gas, emerges from the central pipe. The Reynolds number, based on mean exit velocity and central pipe diameter, is $R e=9700$. The composition is (by volume): $81.29 \% \mathrm{CH}_{4}, 2.87 \% \mathrm{C}_{2} \mathrm{H}_{6}, 0.38 \% \mathrm{C}_{3} \mathrm{H}_{8}, 0.15 \% \mathrm{C}_{4} \mathrm{H}_{10}, 0.04 \% \mathrm{C}_{5} \mathrm{H}_{12}, 0.05 \%$ $\mathrm{C}_{6} \mathrm{H}_{14}, 14.32 \% \mathrm{~N}_{2}, 0.01 \% \mathrm{O}_{2}$ and $0.89 \% \mathrm{CO}_{2}$. For the simulations, the fuel is replaced by a mixture of pure methane and nitrogen with the same caloric value (85.3\% $\mathrm{CH}_{4}$ and $14.7 \% \mathrm{~N}_{2}$ by volume), so that the $\mathrm{C}_{1}$ skeletal scheme [16] can be used. The stoichiometric mixture fraction is $\xi_{\text {st }}=0.070$. Around the central jet, a primary air annulus supplies the air for combustion. A secondary air stream surrounds the burner head. The pilot flames, emerging from 12 small separate holes (diameter $0.5 \mathrm{~mm}$ ), positioned on a circle with radius $=3.5 \mathrm{~mm}$, prevent flame lift-off. The pilot flames consist of an acetylene/hydrogen/air pre-mixture

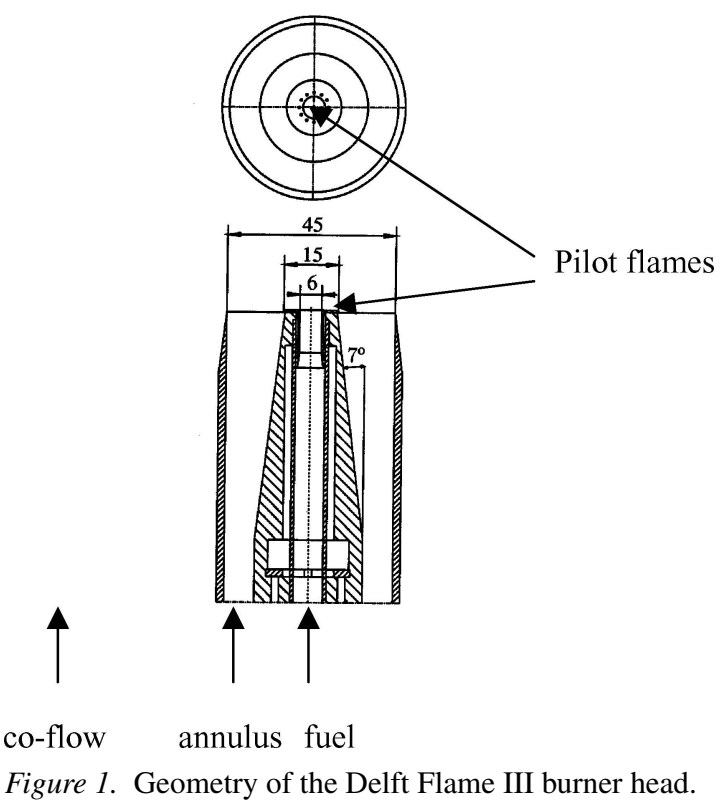


Table I. Experimental set-up: quantitative data.

\begin{tabular}{ll}
\hline Quantity & Value \\
\hline$U_{\text {fuel }}$ & $21.9 \mathrm{~m} / \mathrm{s}$ \\
$T_{\text {fuel }}$ & $295 \mathrm{~K}$ \\
$R e_{\text {fuel }}$ & 9700 \\
$U_{\text {annulus }}$ & $4.4 \mathrm{~m} / \mathrm{s}$ \\
$T_{\text {annulus }}$ & $295 \mathrm{~K}$ \\
$R e_{\text {annulus }}$ & 8800 \\
$U_{\text {coflow }}$ & $0.4 \mathrm{~m} / \mathrm{s}$ \\
$T_{\text {coflow }}$ & $295 \mathrm{~K}$ \\
\hline
\end{tabular}

with equivalence ratio $\Phi=1.4$. The $\mathrm{C} / \mathrm{H}$ ratio equals the ratio of the Dutch natural gas. The heat release rate is about $1 \%$ of the total thermal power. Table I provides additional quantitative experimental information.

Specific nozzle features are the contraction of the central fuel pipe from a diameter of $8 \mathrm{~mm}$ to a diameter of $6 \mathrm{~mm}$ at the nozzle exit, the conical shape of the primary air annulus and the 12 separate holes for the pilot flames. The central constriction is $16 \mathrm{~mm}$ upstream of the nozzle exit. The conical shape of the annulus extends to $60 \mathrm{~mm}$ inside the nozzle: the annulus inner diameter decreases from 30 to $15 \mathrm{~mm}$, with an angle of $7^{\circ}$. The treatment of the pilot flames is described in Section 3.4.

The consequence of the constriction and the conical shape is that the flows, emerging at the nozzle exit, are not fully developed. Therefore, a separate simulation has been performed, in order to obtain reliable profiles at the nozzle exit, which are then used as inlet boundary conditions for the actual flame simulations. The procedure is described in Section 4.

\section{Model Description}

\subsection{TURBULENCE MODEL}

The complete rationale behind the different turbulence model aspects is found in [9-12]. The major aspects are found in the appendix. Here, only the expressions are summarized. Averaging symbols are omitted, except when they are necessary for clarity.

A non-linear, but first order, expression for the turbulent stresses in terms of the local mean strain rate and vorticity tensors (5), is applied, which is sufficient to obtain accurate results for a wide variety of flow types [13]:

$$
-\bar{\rho} \widetilde{v}_{\mathrm{i}}^{\prime \prime} v_{\mathrm{j}}^{\prime \prime}=2 \mu_{\mathrm{t}} S_{\mathrm{ij}}-\frac{2}{3} \rho k \delta_{\mathrm{ij}} .
$$

More important for the test case under study are the (steady-state) transport equations for the turbulence quantities, particularly for the turbulent dissipation 
rate:

$$
\left\{\begin{aligned}
\frac{\partial}{\partial x_{\mathrm{m}}}\left(\rho k v_{\mathrm{m}}\right)= & P_{\mathrm{k}}-\rho \varepsilon+\frac{\partial}{\partial x_{\mathrm{m}}}\left[\left(\mu+\frac{\mu_{\mathrm{t}}}{\sigma_{\mathrm{k}}}\right) \frac{\partial k}{\partial x_{\mathrm{m}}}\right] \\
\frac{\partial}{\partial x_{\mathrm{m}}}\left(\rho \varepsilon v_{\mathrm{m}}\right)= & \left(1-f_{\mathrm{R}_{\mathrm{y}}}\right) c_{\varepsilon 1} \frac{P_{\mathrm{k}}}{\tau_{\mathrm{t}}}+f_{\mathrm{R}_{\mathrm{y}}} C_{1} \rho S \varepsilon-c_{\varepsilon 2} f_{2} \rho \frac{\varepsilon}{\tau_{\mathrm{t}}} \\
& +\frac{\partial}{\partial x_{\mathrm{m}}}\left[\left(\mu+\frac{\mu_{\mathrm{t}}}{\sigma_{\varepsilon}}\right) \frac{\partial \varepsilon}{\partial x_{\mathrm{m}}}\right]+E
\end{aligned}\right.
$$

The source term in the $\varepsilon$ transport equation is a blending of the 'traditional' source term (i.e. the turbulent kinetic energy production term, divided by the turbulence time scale) in the neighborhood of solid boundaries and a source term, derived from a modeled enstrophy transport equation [14]. This second source term part ensures that the well-known plane jet - round jet anomaly disappears [13, 14], while the first part avoids the strong dependence on the inlet boundary conditions and the irregular behavior of the second source term part near solid boundaries.

Important to note is that none of the turbulence model parameters have been tuned for the test case in this paper.

The boundary conditions at a solid boundary are $k=0$ and $\varepsilon=2 \frac{\mu}{\rho}(\partial \sqrt{k} / \partial n)^{2}$. Table II provides an overview of the different model constants.

\subsection{TURBULENCE - CHEMISTRY INTERACTION MODEL}

For the PPDF calculations, $\beta$-PDFs are used, with the mixture fraction as conserved scalar. The (steady-state) transport equations for the mean mixture fraction $\tilde{\xi}$ and its variance $g=\widetilde{\xi^{\prime \prime 2}}$ are standard:

$$
\begin{aligned}
& \frac{\partial\left(\rho \xi v_{\mathrm{m}}\right)}{\partial x_{\mathrm{m}}}=\frac{\partial}{\partial x_{\mathrm{m}}}\left[\frac{\mu_{\mathrm{t}}}{\sigma_{\xi}} \frac{\partial \xi}{\partial x_{\mathrm{m}}}\right] \\
& \frac{\partial\left(\rho g v_{\mathrm{m}}\right)}{\partial x_{\mathrm{m}}}=\frac{\partial}{\partial x_{\mathrm{m}}}\left[\frac{\mu_{\mathrm{t}}}{\sigma_{\mathrm{g}}} \frac{\partial g}{\partial x_{\mathrm{m}}}\right]+2 \frac{\mu_{\mathrm{t}}}{\sigma_{\xi}} \frac{\partial \xi}{\partial x_{\mathrm{m}}} \frac{\partial \xi}{\partial x_{\mathrm{m}}}-\rho \chi
\end{aligned}
$$

Note that the molecular diffusion is neglected in Equations (3) and (4): only the turbulent diffusion, modeled by the linear gradient diffusion hypothesis, is accounted for. The turbulent Schmidt numbers are $\sigma_{\xi}=\sigma_{\mathrm{g}}=0.85$, which is somewhat higher than the standard value of 0.7. This was explained in [10] for a round jet flame: near the axis, the eddy viscosity is higher with the present turbulence model than what is obtained with the standard $k-\varepsilon$ model. As a consequence, mixture fraction spreading rate is over-estimated with the standard value $\sigma_{\xi}=\sigma_{\mathrm{g}}=0.7$, despite a correct

Table II. Overview of turbulence model parameters.

\begin{tabular}{llllll}
\hline Parameter & $c_{\varepsilon 1}$ & $c_{\varepsilon 2}$ & $\mathrm{C}_{1}$ & $\sigma_{k}$ & $\sigma_{\varepsilon}$ \\
Value & 1.44 & $\max \left(1.9 f_{R_{\mathrm{y}}} ; 1.83+\frac{0.075 \Omega \tau_{\mathrm{t}}}{1+S^{2} \tau_{\mathrm{t}}^{2}}\right)$ & $\max \left(0.43 ; \frac{S \tau_{\mathrm{t}}}{5+S \tau_{\mathrm{t}}}\right)$ & 1.0 & 1.2 \\
\hline
\end{tabular}


spreading rate for the underlying flow field. The applied value $\sigma_{\xi}=\sigma_{\mathrm{g}}=0.85$ is not extreme: values up to 0.9 are encountered in the literature.

The mean scalar dissipation rate is modeled as:

$$
\chi=2 g \frac{\varepsilon}{k}
$$

For the TPDF simulations, the transport equation of the mass density function is solved:

$$
\frac{\partial F}{\partial t}+\frac{\partial}{\partial x_{\mathrm{j}}}\left(\tilde{v}_{\mathrm{j}} F\right)+\frac{\partial}{\partial \psi_{\mathrm{k}}}\left(S_{\mathrm{k}} F\right)=\frac{\partial}{\partial x_{\mathrm{j}}}\left(\Gamma_{\mathrm{t}} \frac{\partial \tilde{f}}{\partial x_{\mathrm{j}}}\right)+\frac{\partial}{\partial \psi_{\mathrm{k}}}\left(\left\langle\frac{1}{\rho} \frac{\partial J_{\mathrm{i}, \mathrm{k}}}{\partial x_{\mathrm{i}}} \mid \psi\right\rangle F\right)
$$

Here, $F(\psi ; x, t)=\langle\rho\rangle \tilde{f}(\psi ; x, t)$ is the mass density function, where $\tilde{f}(\psi ; x, t)$ is the Favre PDF. The velocity components are not included as density variables in the PDF: the transported scalar PDF approach is used, as described in Chapter 7 of [15].

A spatially second order accurate Lagrangian method is used to solve Equation (14). The "mid-point rule" is applied in order to achieve second order accuracy for time integration of particle property evolution. A first (half) time step brings the particle to a midpoint position:

$$
X_{\mathrm{i}}^{n+1 / 2}=X_{\mathrm{i}}^{n}+0.5 \tilde{v}_{\mathrm{i}}^{n} \Delta t,
$$

where the mean fields are interpolated for the particle property evolution, with $\tilde{v}_{\mathrm{i}}$ the i-component of the Favre mean fluid velocity vector at the particle position. Diffusion and reaction sub-processes are subsequently treated, using the mean field values interpolated at the mid-point. Finally, a second (full) time step brings the particles to their final position (from their initial position) using the mid-point mean field values:

$$
X_{\mathrm{i}}^{n+1}=X_{\mathrm{i}}^{n+1 / 2}+\Delta t\left(\tilde{v}_{\mathrm{i}}^{n+1 / 2}-0.5 \tilde{v}_{\mathrm{i}}^{n}+\frac{1}{\langle\rho\rangle} \frac{\partial \Gamma_{\mathrm{t}}}{\partial x_{\mathrm{i}}}+\xi_{\mathrm{i}} \sqrt{\frac{2 \Gamma_{\mathrm{t}}}{\langle\rho\rangle \Delta t}}\right)
$$

with $\xi_{\mathrm{i}}$ a standardized Gaussian random variable. In this procedure, the time step $\Delta t$ is locally determined from $\Delta t=\min \left(\Delta t_{\mathrm{c}} ; \Delta t_{\mathrm{d}} ; \Delta t_{\mathrm{m}}\right)$, with $\Delta t_{\mathrm{c}}=0.5 A^{0.5} /|\tilde{v}|$, $\Delta t_{\mathrm{d}}=0.5\langle\rho\rangle A / \Gamma_{\mathrm{t}}$ and $\Delta t_{\mathrm{m}}=0.5 \mathrm{k} / \varepsilon$. In the last expressions, $A$ is the cell surface area, $\Gamma_{\mathrm{t}}=\mu_{\mathrm{t}} / \sigma_{\xi}$ the turbulent diffusivity and cell properties are used. Note that these expressions are similar to the ones reported in [19], except for the choice 0.5 (in [19], 0.4 is used in $\Delta t_{\mathrm{c}}$ and $0.2 \mathrm{in} \Delta t_{\mathrm{m}}$; the restriction $\Delta t_{\mathrm{d}}$ is replaced by a global limitation). The use of local time steps accelerates the convergence by an order of magnitude and significantly improves the robustness of the hybrid method. In [18], the proof is given that local time stepping may be used for transported scalar PDF.

The number of particles per cell is set to 100 , which is sufficient with respect to the statistical error [18]. Statistical error is further reduced through averaging over the latest 50 iterations [19].

An important term in (14) is the final term, expressing transport in sample space due to micro-mixing. Here, the EMST model [17] is applied, with the standard 
value for the model constant $C_{\phi}=2$. In [18], different micro-mixing models, such as the IEM [20] and modified Curl's [21] coalescence-dispersion method (CD), are compared, as well as different values for the model constant $C_{\phi}$. It is illustrated in [18] that the EMST model is the only micro-mixing that yields an attached flame when the experimental pilot flame power is imposed (see Section 3.4): the IEM model leads to global flame extinction and the CD model yields a lifted flame, in contrast to the experimental observations.

The numerical observations for the different micro-mixing models' behavior are in line with [2], where a lifted flame was obtained when combining ILDM with CD and $C_{\phi}=2$ (an attached flame was only obtained when burning laminar flamelet conditions were imposed in a region near the nozzle exit and $\mathrm{C}_{\phi}$ was increased from 2 to 4). They are also in line with the conclusions of [22], where it is stated that the EMST micro-mixing model, compared to the IEM and CD mixing models, has the strongest resistance to extinction when the mixture fraction variance is high. This is the case in the region near the burner nozzle.

\subsection{CHEMISTRY MODEL}

Three different chemistry models are compared. Evidently, the applied model must be sufficiently accurate in order not to destroy the quality of the obtained flow field predictions. For the PPDF simulations, the first model is full chemical equilibrium. The main species are (maximum mean mass fraction in the field larger than $10^{-7}$ ): $\mathrm{CH}_{4}, \mathrm{O}_{2}, \mathrm{H}_{2} \mathrm{O}, \mathrm{CO}_{2}, \mathrm{CO}, \mathrm{OH}, \mathrm{H}_{2}, \mathrm{HO}_{2}, \mathrm{O}, \mathrm{H}, \mathrm{NO}, \mathrm{NO}_{2}, \mathrm{C}_{2} \mathrm{H}_{6}, \mathrm{~N}_{2}$. The second model is a steady laminar flamelet model (constant strain rate $a=100 \mathrm{~s}^{-1}$ ), based on the $\mathrm{C}_{1}$ skeletal scheme of [16]. This mechanism consists of 16 species $\left(\mathrm{CH}_{4}\right.$, $\mathrm{O}_{2}, \mathrm{H}_{2} \mathrm{O}, \mathrm{CO}_{2}, \mathrm{CO}, \mathrm{OH}, \mathrm{CH}_{2} \mathrm{O}, \mathrm{H}_{2}, \mathrm{HO}_{2}, \mathrm{H}_{2} \mathrm{O}_{2}, \mathrm{O}, \mathrm{H}, \mathrm{HCO}, \mathrm{CH}_{3}, \mathrm{CH}_{3} \mathrm{O}, \mathrm{N}_{2}$ ) and 41 reactions.

For the TPDF simulations, the same $\mathrm{C}_{1}$ skeletal scheme of [16] is used.

\subsection{PilOT FlAMES}

As described in Section 2, pilot flames emerge from 12 separate holes, in order to avoid flame lift-off. In order to drastically reduce computing time and memory requirements, the simulations are performed under the assumption of axisymmetry, so that a rectangular grid is sufficient. Evidently, the pilot flames then require special attention.

Important to note is that their mass flow rate $\left(\approx 2.3 \times 10^{-5} \mathrm{~kg} / \mathrm{s}\right)$ and momentum flow rate $\left(\approx 2.3 \times 10^{-3} \mathrm{~N}\right)$ are small, compared to the fuel $\left(\approx 4.9 \times 10^{-4} \mathrm{~kg} / \mathrm{s}\right.$ and $0.01 \mathrm{~N})$ and air $\left(\approx 7.6 \times 10^{-3} \mathrm{~kg} / \mathrm{s}\right.$ and $\left.3.4 \times 10^{-2} \mathrm{~N}\right)$ inlets. Also the pilot flames' thermal power is small (around $200 \mathrm{~W}$, i.e. around $1 \%$ of the total thermal power). As a consequence, neglecting the pilot flames is a realistic approximation, as long as the flame ignites. Therefore they were omitted in the PPDF simulations. It is illustrated that this is justified (see later). 
For the TPDF simulations, on the other hand, the flame numerically extinguishes unless the thermal power (about $200 \mathrm{~W}$ ) of the pilot flames is supplied. It is indeed more important to supply the heat, rather than the mass flow rate of the pilot flames. Here, the thermal power is simply added into the (steady-state) mean enthalpy transport equation as a volumetric source term in a well-defined region:

$$
\frac{\partial\left(\rho h v_{\mathrm{m}}\right)}{\partial x_{\mathrm{m}}}=\frac{\partial}{\partial x_{\mathrm{m}}}\left[\frac{\mu_{\mathrm{t}}}{\operatorname{Pr}_{\mathrm{t}}} \frac{\partial h}{\partial x_{\mathrm{m}}}\right]+Q
$$

Two different source term definitions are compared in Section 3.4:

$$
Q_{1}: 10 \mathrm{~mm}<x<20 \mathrm{~mm} ; 6 \mathrm{~mm}<r<8 \mathrm{~mm} ; Q=2.310^{8} \mathrm{~W} / \mathrm{m}^{3}
$$

and

$$
Q_{2}: 0 \mathrm{~mm}<x<20 \mathrm{~mm} ; 3.5 \mathrm{~mm}<r<7 \mathrm{~mm} ; Q=8.710^{7} \mathrm{~W} / \mathrm{m}^{3}
$$

The total supplied thermal power is about $200 \mathrm{~W}$ for both sources. The rationale behind $Q_{1}$ is that the highest measured temperatures at $x=25 \mathrm{~mm}$ are positioned between $r=6 \mathrm{~mm}$ and $r=8 \mathrm{~mm}$. Note that it makes sense that this range of radii is further away from the axis than the ring in the nozzle exit (with radius $=3.5 \mathrm{~mm}$ ): the central jet spreads, so that the pilot flames are pushed away from the axis. The reason to start the pilot flame region $10 \mathrm{~mm}$ downstream of the nozzle exit, is that the mixing of the pilot flames and the central jet is much better than at the burner head itself, so that the assumption of axisymmetry is more plausible. However, as will be explained later, this leads to large deviations in the experimental measurement region, due to large deviations in the flow field. It is explained in Section 5.3 that this problem is overcome when the pilot flame region starts at the burner head. Consequently, the inner radius has been chosen equal to the radius of the pilot flames (i.e. $3.5 \mathrm{~mm}$ ). The outer radius was chosen as $7 \mathrm{~mm}$ and axially the region extends to $x=20 \mathrm{~mm}$. Variations of the magnitude of this region are possible, but it must start at $x=0 \mathrm{~mm}$.

To conclude this discussion, it is noted that injection of hot pilot gases seems a better option at first sight, but this is not straightforward. In the simulations, the area of the 12 separate pilot holes is 'smeared' over an annular area with width equal to one cell size, i.e. $0.5 \mathrm{~mm}$. It is not tractable to make the annular area equal to the experimental area of the 12 holes, since this results in very fine cells. As a result of the smearing with the present grid, the experimental pilot mass and momentum flow rates cannot be correctly imposed at the same time. When the experimental mass flow rate is imposed, the pilot flames' momentum is too small for the flame to ignite. When the correct momentum is imposed, the mass flow rate is too high and the flow field is disturbed.

Enforcing chemical equilibrium in a certain region near the pilot flame zone is also a possibility [2], but this turned out to be impossible to do within the commercial package FLUENT, used for this work. 
A final remark concerns the axisymmetric representation of the threedimensional pilot flames, which may affect flame stabilization. The spontaneous emission of the pilot flames shows that the three-dimensional effects already disappear after a few millimeters [3]. The product streams of the individual pilot flames then combine to form a cylindrically symmetric stream, which strongly suggests that the three-dimensionality is not critical for flame stabilization. Another consideration is the fact that the stabilization is obtained by a combination of a pilot flame and a bluff-body effect (behind the rim between the central fuel jet and the primary air annulus). In order to have a good representation of the bluff body effect and the entrainment of air by the fuel jet, it is again important to represent the pilot flames by addition of heat, right at the rim and not further downstream. A separate three-dimensional pilot flame flow calculation may be a more rigorous way to model their effect, but some averaging procedure will remain inevitable for the final axisymmetric calculations.

\section{Construction of Inlet Boundary Conditions}

In order to obtain reliable inlet profiles for the flame simulations, separate calculations have been performed inside the burner head, on fine grids. For the constriction in the central pipe, the grid consists of $100 \times 100$ cells. At the inlet, positioned at $x=-25 \mathrm{~mm}$, a fully developed pipe flow is imposed. This fully developed flow is, on its turn, separately computed a priori on a fine grid in a pipe with diameter equal to $8 \mathrm{~mm}$. The mean axial velocity, turbulent kinetic energy and turbulent dissipation rate, obtained at the nozzle exit, are imposed as inlet boundary conditions for the flame simulations. The wall $y^{+}$value is always between 0.5 and 0.9 in all mentioned simulations.

The primary air annulus has a conical shape. Here, the grid consists of $140 \times$ 100 cells. At the inlet, positioned at $x=-70 \mathrm{~mm}$, a separately computed fully developed annulus flow is imposed. The wall $y^{+}$value, both on the inner and the outer radius, is always between 0.5 and 1 . The mean axial and (non-zero) radial velocity components, as well as turbulent kinetic energy and dissipation rate at the nozzle exit, are imposed as inlet boundary conditions for the flame computations.

For the co-flow (or secondary) air stream, uniform profiles are imposed [5]: $U=0.4 \mathrm{~m} / \mathrm{s}, k=6.10^{-4} \mathrm{~m}^{2} / \mathrm{s}^{2}$ and $\varepsilon=5.4 \times 10^{-5} \mathrm{~m}^{2} / \mathrm{s}^{3}$.

In previous work (e.g. [2]), less attention was given to the generation of the inlet boundary conditions. There, uniform profiles were imposed for mean axial velocity $(U=21.9 \mathrm{~m} / \mathrm{s})$, turbulent kinetic energy $\left(k=2.2 \mathrm{~m}^{2} / \mathrm{s}^{2}\right)$ and dissipation rate $\left(\varepsilon=500 \mathrm{~m}^{2} / \mathrm{s}^{3}\right)$ for the central fuel jet. For the primary air annulus, a fully developed annulus flow was imposed, with zero mean radial velocity. For the co-flow, flat profiles were used $(U=0.4 \mathrm{~m} / \mathrm{s}$, turbulence intensity $=5 \%)$. 


\section{Results}

All results have been obtained with the commercial software package FLUENT, versions 6.1 and 6.2. The basic computational grid consists of $100 \times 60$ cells and extends from $x=0 D$ to $x=100 D$ and from $r=0 D$ to $r=60 D$, with $D=6 \mathrm{~mm}$ the central pipe diameter. Grid independence of the results has been verified.

\subsection{INFLUENCE OF THE TURBULENCE MODEL}

\subsubsection{Cold Flow}

In order to verify the turbulence model's quality, cold flow results are presented first. The inlet velocities are again found in Table I. Inclusion or neglect of the 'pilots' does not visibly affect the results obtained (not shown).

In Figure 2, radial profiles of the mean and rms values of the axial velocity component are presented at five different axial positions: $x=50 \mathrm{~mm} ; x=100 \mathrm{~mm}$; $x=150 \mathrm{~mm} ; x=200 \mathrm{~mm} ; x=250 \mathrm{~mm}$. The present model is in reasonable agreement with the experimental data [23], although none of the model parameters have been tuned for the specific test case under study. With the standard $k-\varepsilon$ model [24], the well-known deficiency for a round jet is observed: too rapid mean axial velocity decay on the axis and too large spreading rate. This goes hand in hand with excessive turbulent kinetic energy levels, expressed here in the profiles of the rms value of the mean axial velocity fluctuations. Increasing the model constant $\mathrm{c}_{\varepsilon 1}$ from its standard value 1.44 to 1.6 corrects for the round jet's spreading rate. Indeed, agreement with experimental data improves, despite an over-estimation near the axis. This also causes an over-estimation of the velocity fluctuations in that region.

\subsubsection{Reacting Flow}

The numerical set-up for this set of calculations is as follows. The inlet boundary conditions are constructed with the procedure, described in Section 4. The PPDF method is applied, in combination with chemical equilibrium. The pilot flames are omitted.

Figure 3 shows contours of mean temperature, obtained by the standard $k-$ $\varepsilon$ model [24], with the adjusted model constant $c_{\varepsilon 1}=1.6$ and with the present non-linear turbulence model. Clearly, the flame length increases when the current model is used, which is in line with the results of [9]. When $c_{\varepsilon 1}=1.6$ is used in the $k-\varepsilon$ model, the flame length is even larger. The experimental data are restricted to a region, close to the burner head: the most downstream distance where data are available, is $x=250 \mathrm{~mm}$. Consequently, no hard statement can be made on the accuracy of the flame length. Still, the profiles of Figure 5 indicate that, as in [9], the flame length is slightly over-estimated with the current model, while it is seriously under-estimated with the standard $k-\varepsilon$ model due to 

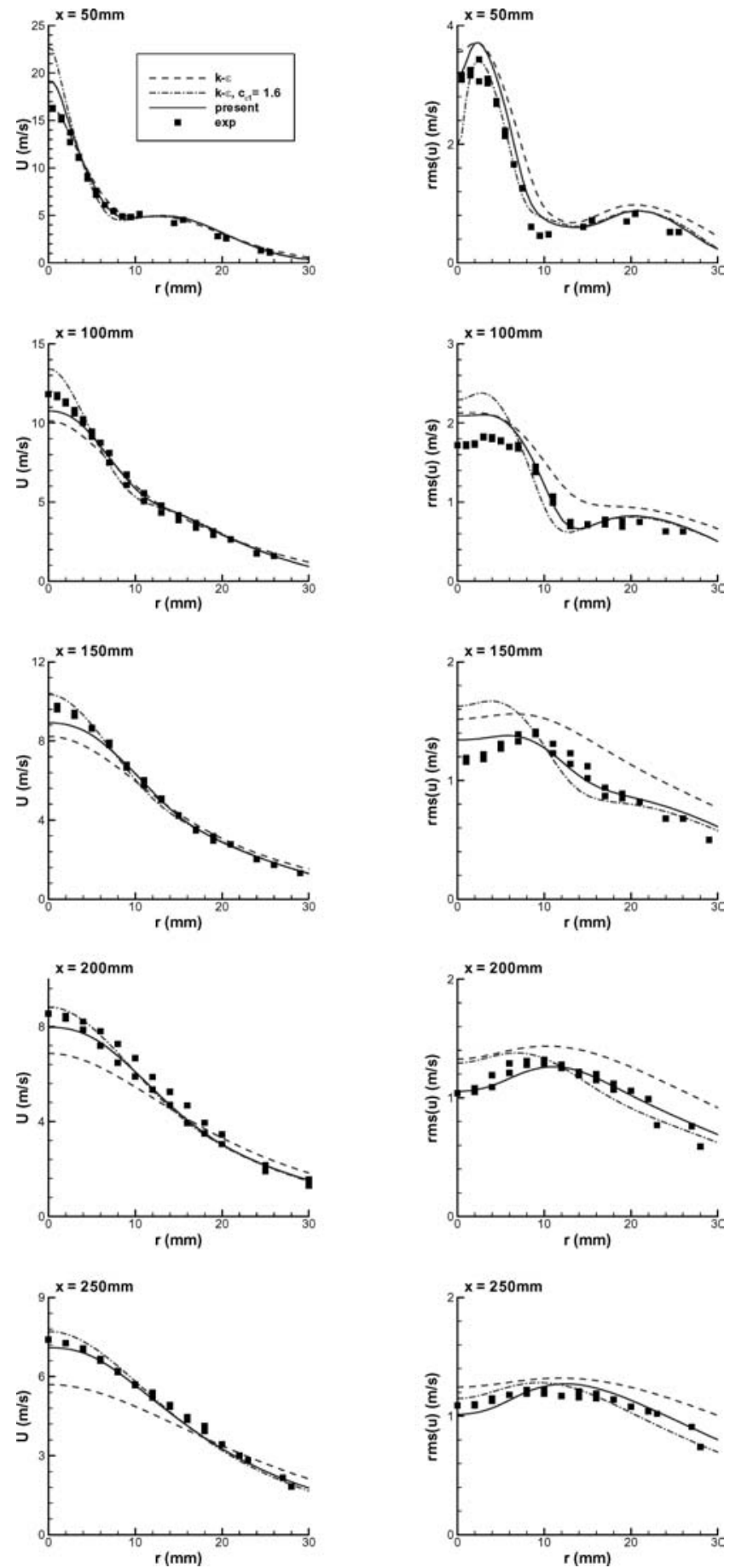

Figure 2. Radial profiles of mean axial velocity and rms of the axial velocity fluctuations at different axial positions (cold flow). 

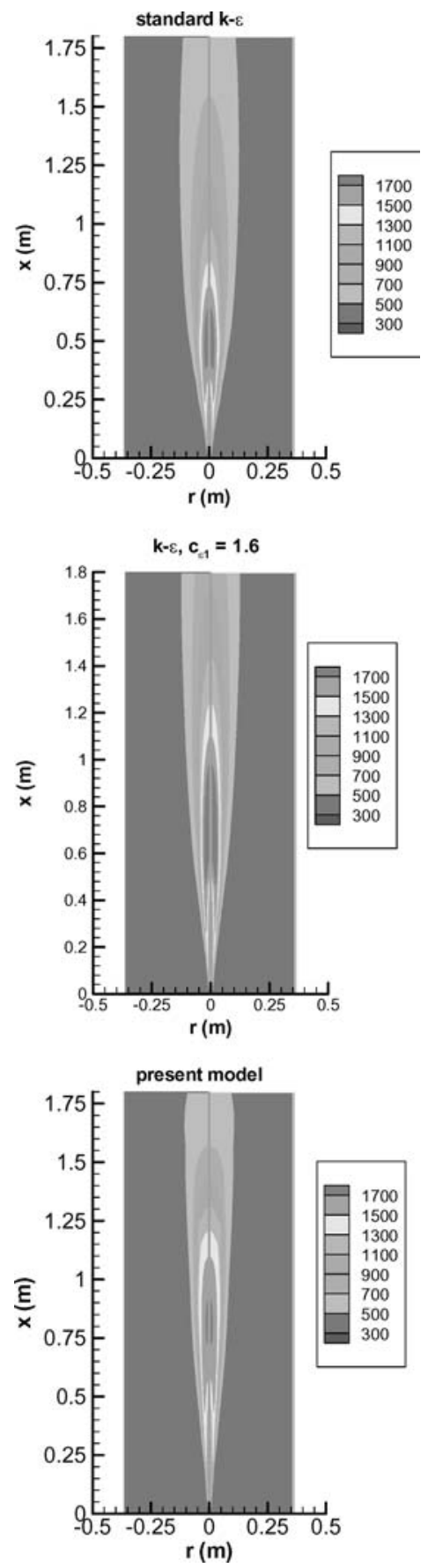

Figure 3. Contours of mean temperature: comparison of turbulence models. 
excessive turbulent mixing. This is clearly observed in the much larger spreading rate.

Figures 4 and 5 confirm the excessive spreading rate with the standard $k-\varepsilon$ model for radial profiles at the five different axial positions. In Figure 4, it is observed that the level of turbulent kinetic energy is much too high with the standard $k-$ $\varepsilon$ model near the burner. This results in too large values for the eddy viscosity and turbulent shear stresses, so that the central jet decelerates far too quickly (see Section 5.4 for a more detailed discussion on this) and spreads too much. Further downstream, the level of turbulent kinetic energy seems in better agreement with the experimental data, but this is mainly due to the under-estimated mean velocity level: the turbulence intensity and the spreading rate remain over-estimated. With the present model, the shapes of the turbulent kinetic energy profiles are in good agreement with the experimental data, but the global level is slightly too high. The mean velocity profiles are in reasonable agreement with the experimental data, too. In particular, the shapes (and spreading rate) are very well reproduced. The global under-estimation for the downstream positions is due to the under-estimation of the mean temperature (Figure 5): mean density is over-estimated, so that, due to conservation of mass, the global mean axial velocity is too low. In [2], similar flow field results were obtained as with the present model, using the standard $k-\varepsilon$ model with a round jet correction [25]. This model is, however, not as general to apply with confidence as the present model, which has been applied to a variety of flow types without test case dependent model parameter tuning [9-12]. It is recalled here that the correct jet spreading rate is mainly due to the source term in the $\varepsilon$ transport equation (9) and that there is no plane jet - round jet anomaly with the present model.

Figure 5 shows the mean mixture fraction, the rms value of its fluctuations and the mean temperature. The mixture fraction is determined from the experimental data for the different species, according to Bilger's formula [26]:

$$
\xi=\frac{2 \frac{Y_{\mathrm{C}}-Y_{\mathrm{C}, \mathrm{o}}}{W_{\mathrm{C}}}+\frac{Y_{\mathrm{H}}-Y_{\mathrm{H}, \mathrm{o}}}{2 W_{\mathrm{H}}}-\frac{Y_{\mathrm{O}}-Y_{\mathrm{O}, \mathrm{o}}}{W_{\mathrm{O}}}}{2 \frac{Y_{\mathrm{C}, \mathrm{f}}-Y_{\mathrm{C}, \mathrm{o}}}{W_{\mathrm{C}}}+\frac{Y_{\mathrm{H}, \mathrm{f}}-Y_{\mathrm{H}, \mathrm{o}}}{2 W_{\mathrm{H}}}-\frac{Y_{\mathrm{O}, \mathrm{f}}-Y_{\mathrm{O}, \mathrm{o}}}{W_{\mathrm{O}}}}
$$

where the subscripts ' $o$ ' and ' $\mathrm{f}$ ' denote oxidizer and fuel, respectively.

The mean mixture fraction evolution is governed by convection-diffusion equation (3), so that it is not surprising that the spreading rate is well predicted with the present model. It is noteworthy that the value $\sigma_{\xi}=0.85$, determined in [10], is higher than the standard value $\sigma_{\xi}=0.7$ (but still within the generally accepted range 0.7-0.9), because the eddy viscosity near the axis is higher than what is obtained with the standard $k-\varepsilon$ model. This is due to the higher value of $c_{\mu}$ (Equation (A.5)) near the axis. Consequently, the standard value $\sigma_{\xi}=0.7$ leads to excessive spreading rates for the mean mixture fraction. A complete discussion has already been given in [10]. 

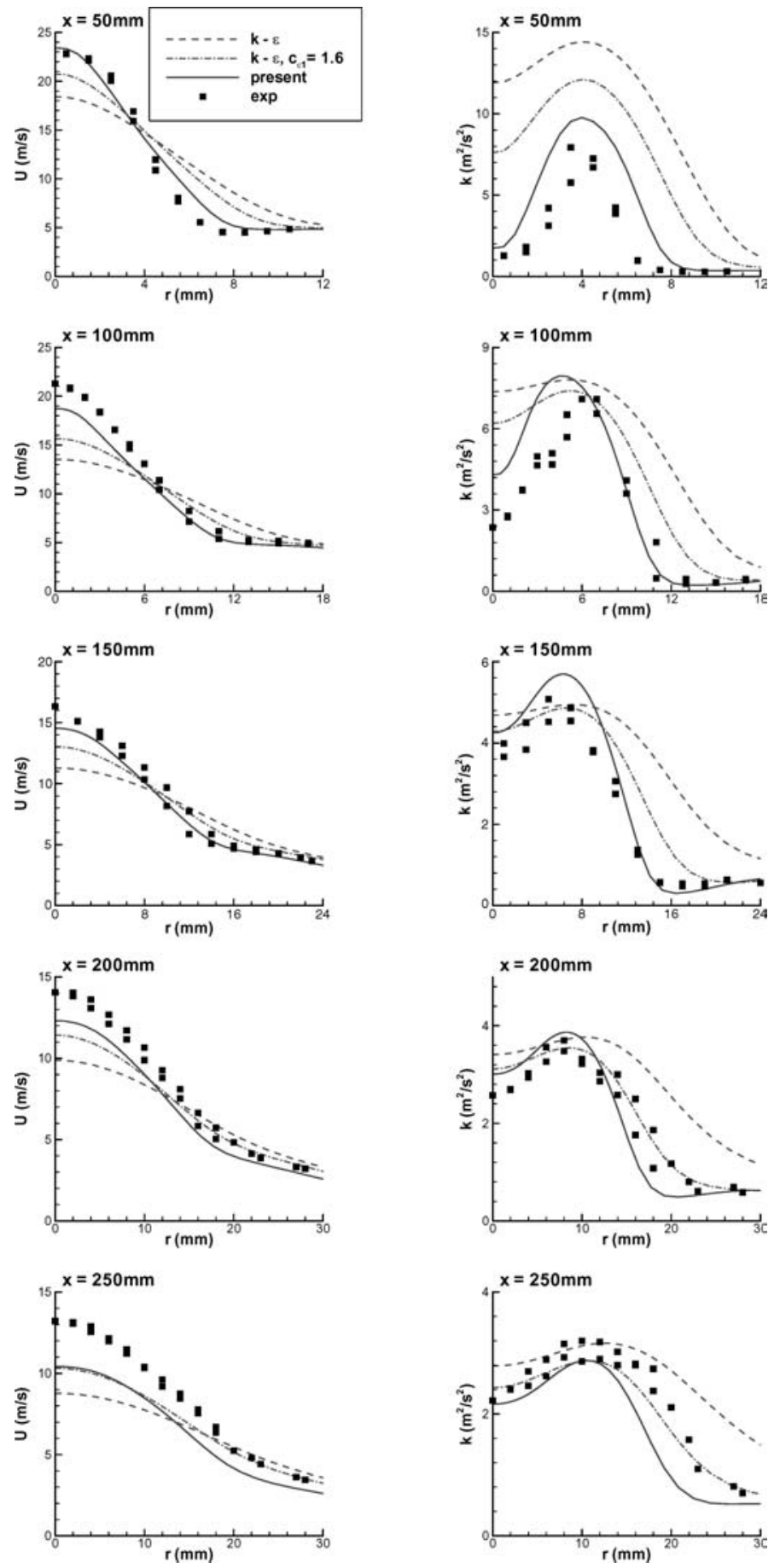

Figure 4. Radial profiles of mean axial velocity and turbulent kinetic energy: comparison of turbulence models. 

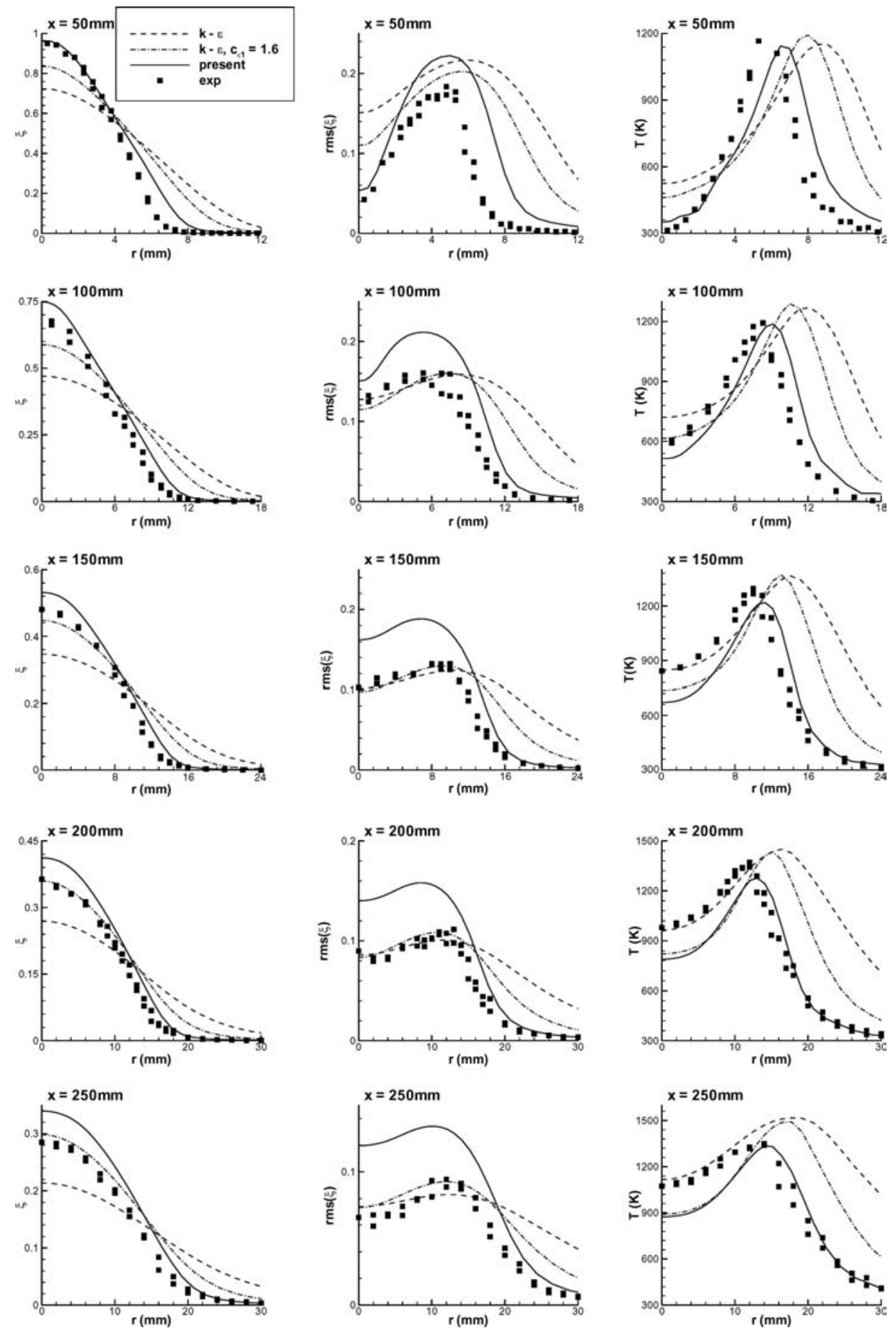

Figure 5. Radial profiles of mean mixture fraction, rms value of mixture fraction fluctuations and mean temperature: comparison of turbulence models. 
It is noted that the mean mixture fraction value is over-estimated near the axis. This remains true at $x=200 \mathrm{~mm}$ and $x=250 \mathrm{~mm}$, despite the under-estimation of mean velocity. The reason is to be found in the under-estimation of the mean temperature. As a consequence, mean density is over-estimated. Assuming the mean density inversely proportional to the mean temperature, it is easily verified that the product $\rho U$ is over-predicted by about $8 \%$, which explains excessive convection at the axis, resulting in over-predicted mean mixture fraction values. It could be expected that this is counter-acted by excessive diffusion, too, due to higher eddy viscosity values (proportional to the mean density). Indeed, the over-prediction is global, but the larger amount of diffusion does not compensate the over-prediction on the axis. In this light, it is also possible that the value $\sigma_{\xi}=0.85$ is somewhat too high for the current test case, but we deliberately avoid test case dependent parameter tuning here.

The shapes of the profiles of the rms value of the mixture fraction fluctuations are in good agreement with the experimental data for all positions with the present model. In particular, the spreading rate is well predicted (in contrast to the standard $k-\varepsilon$ model results). The absolute values are too high, though. The main reason is the over-prediction of the mean mixture fraction, so that the source term in Equation (12), proportional to the square of the mean mixture fraction derivatives, is too high. This over-prediction could easily be remedied by raising the value 2 in Equation (13), but again parameter tuning has been avoided. Moreover, the influence of an error made in the prediction of the mixture fraction variance on e.g. the mean temperature is smaller than an error in the mean mixture fraction in the test case under study.

Finally, the mean temperature profiles again show the correct shape with the present model, with the peak temperature positioned slightly too far away from the axis. The under-prediction in the region between the axis and the peak temperature position is due to two reasons: the lack of accuracy of the applied chemical equilibrium chemistry model, by which temperature is under-predicted on the rich side of stoichiometric mixture fraction $\xi_{\mathrm{st}}=0.070$; and the over-prediction of both mean mixture fraction and its variance, both leading to lower temperatures. On the lean side, agreement with the experiments is good.

\subsection{INFLUENCE OF THE CHEMISTRY MODEL}

In this section, pre-assumed PDF results with chemical equilibrium are compared to what is obtained when the steady laminar flamelet, described in Section 3.3, is applied. The present turbulence model is used for both simulations.

Figure 6 reveals that the differences in the mean axial velocity and turbulent kinetic energy profiles are much smaller than the differences observed in Figure 4, where the different turbulence models were compared. The mean velocity is slightly better predicted with the laminar flamelet model, due to the better agreement for the mean temperature near the axis (Figure 7): the thermal expansion is reproduced 

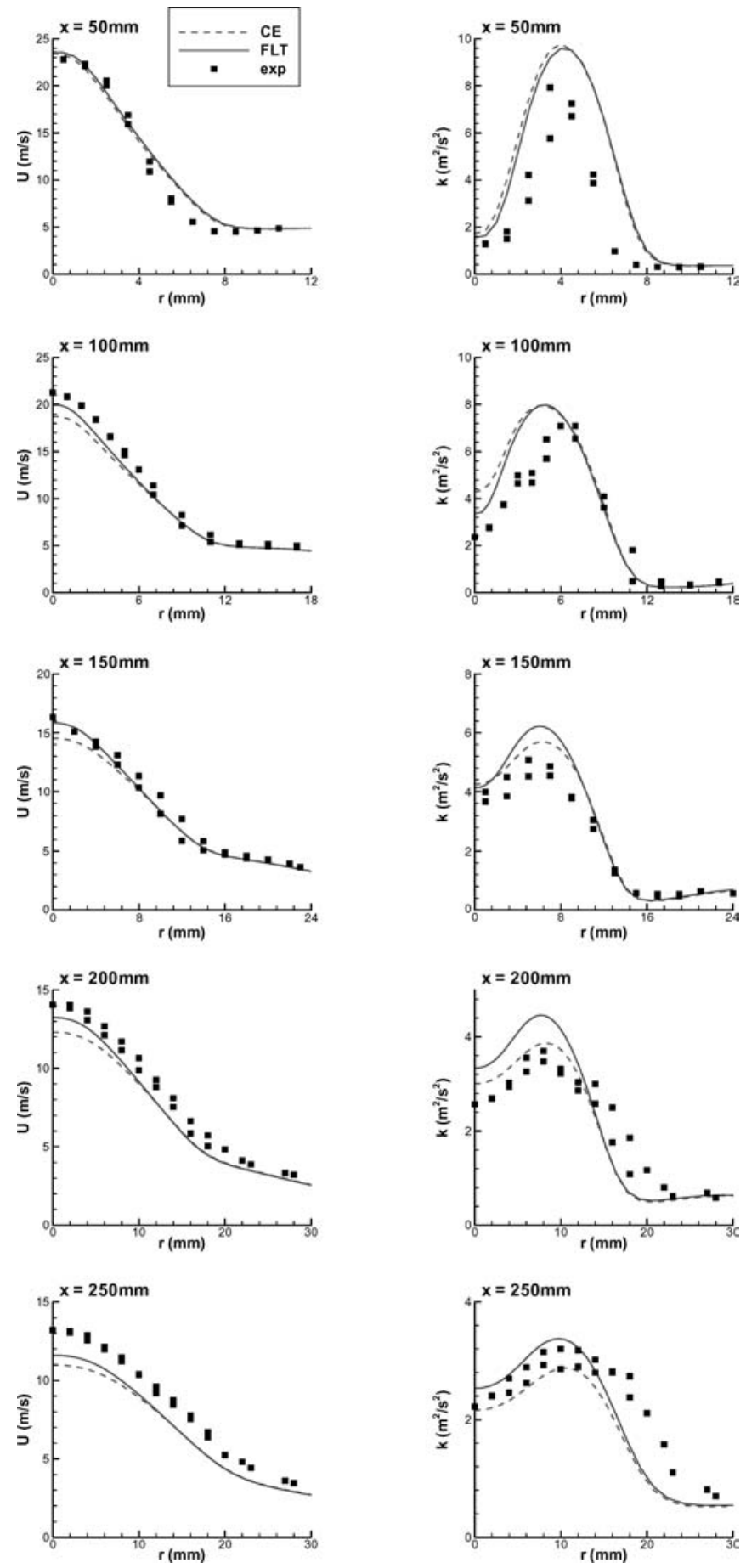

Figure 6. Radial profiles of mean axial velocity and turbulent kinetic energy: comparison of chemistry models (PPDF results). (CE: chemical equilibrium; FLT: laminar flamelet model). 

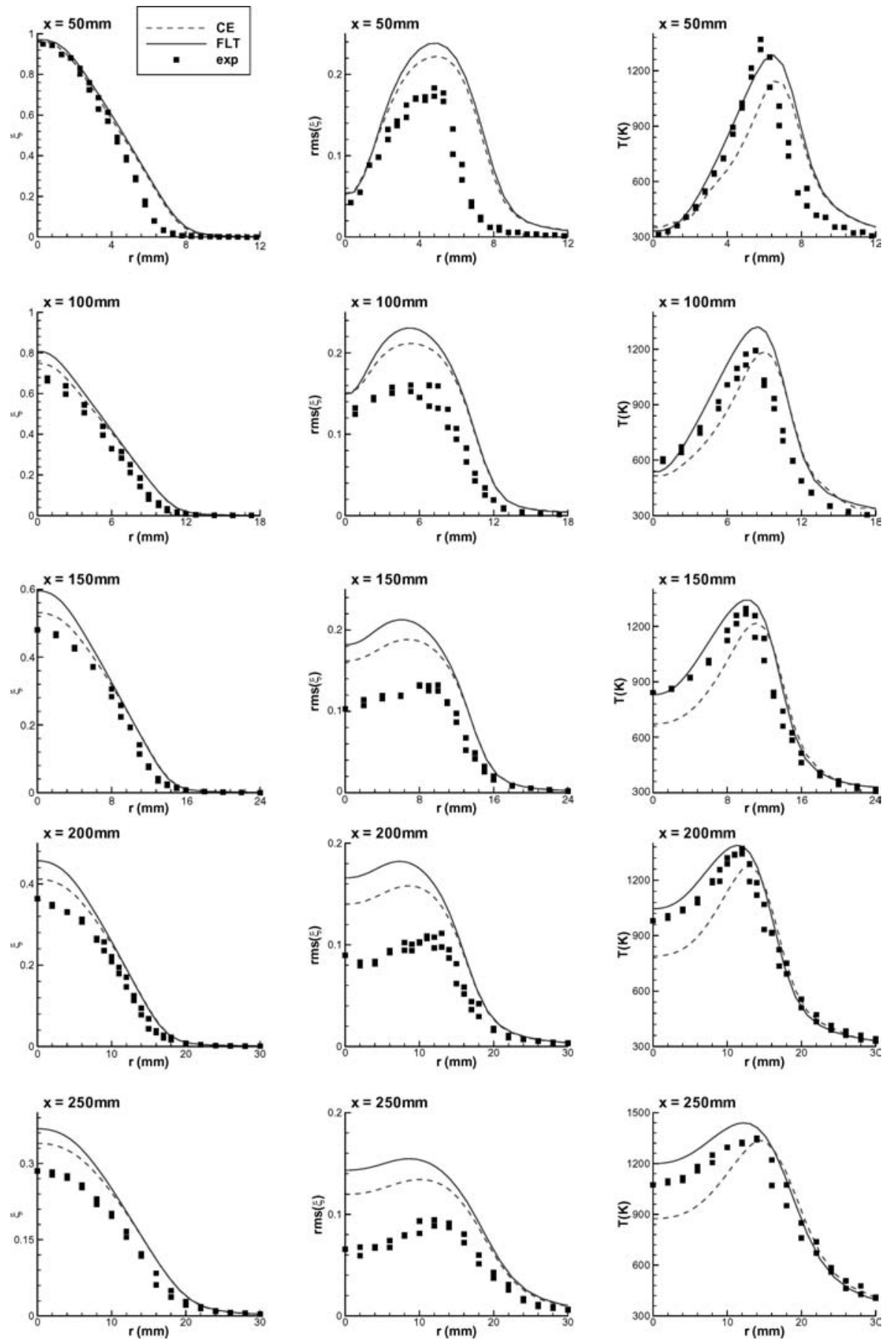

Figure 7. Radial profiles of mean mixture fraction, rms value of mixture fraction fluctuations and mean temperature: comparison of chemistry models (PPDF results). (Legend as in Figure 6). 
more accurately. On the other hand, the turbulent kinetic energy is slightly overestimated due to the higher velocities.

The over-estimation of mean mixture fraction is worse with the flamelet model due to the higher velocities (or rather higher $\rho U$ values near the nozzle) and the history effects in the flow: higher mean mixture fraction values are convected downstream. As a consequence of the higher mean mixture fraction values, the mixture fraction variance is higher, too, as explained above.

For the mean temperature, the results are better with the laminar flamelet model. The shape is better: better agreement near the axis and slightly better prediction of radial position of peak temperature. The latter is due to the fact that the maximum temperature is on the rich side of stoichiometric mixture fraction value, as in [9]. On the other hand, it must be noted that the temperature is over-predicted, despite over-prediction of mixture fraction variance, which should actually lead to lower mean temperatures. A similar remark can be made about the good temperature agreement near the axis despite over-estimation of both mean mixture fraction and its variance. This leads to the conclusion that temperature is over-estimated in the rich region with the laminar flamelet model (while it is under-estimated by the chemical equilibrium model).

\subsection{INFLUENCE OF THE INLET BOUNDARY CONDITIONS}

The impact of the inlet boundary conditions on the global flame shape is small, as illustrated in Figure 8. On the right side of this figure, the temperature contour plots are given when the inlet boundary conditions are constructed with the procedure, described in Section 4. On the left side, the inlet is as in [2]. For both simulations, the turbulence model of Section 3.1 is applied. The flame is slightly longer (hardly visible) with the inlet conditions of [2], but the global shape (e.g. spreading of the flame) is very similar.
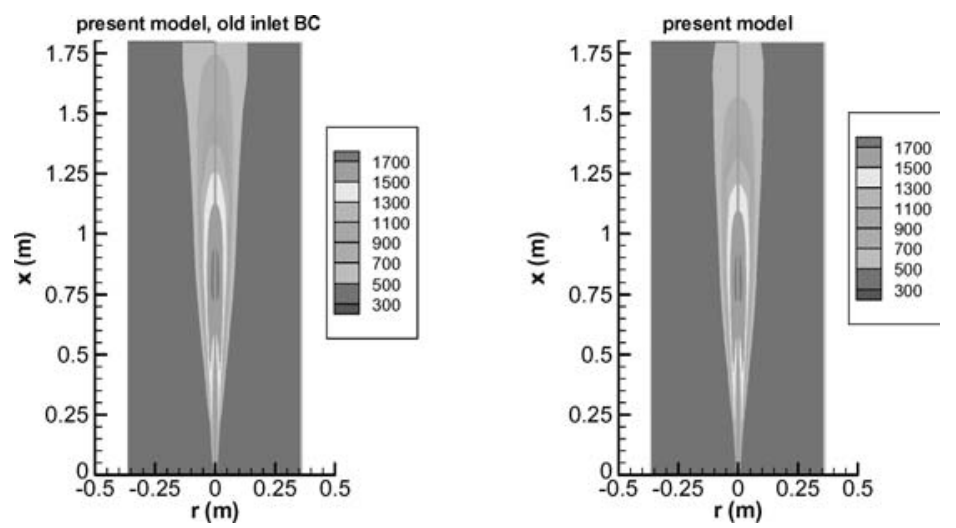

Figure 8. Contours of mean temperature: influence of inlet boundary conditions. 
Indeed, Figures 9 and 10 confirm that there are noticeable differences in the different radial profiles, but the deviations are clearly not as large as observed in Figures 4 and 5, where the choice of the turbulence model was investigated. It is hardly possible to argument that, despite the obviously rigorous approach of the procedure described in Section 4, the global quality of the results improves, compared to the results with the 'simple' inlet boundary conditions of [2]. There is clearly an improvement in the mean velocity field predictions, in particular close to the burner. However, the (more correct) higher velocities go hand in hand with a globally higher level of turbulent kinetic energy. This leads to increased diffusion of mean mixture fraction, so that the profiles of mean mixture fraction almost coincide, independent of the inlet boundary conditions (except close to the burner again). This also results in very comparable mean temperature profiles. The mixture fraction variance is less over-estimated with the inlet BC of [2], because of the lower level of turbulent kinetic energy: the lower eddy-viscosity leads to a smaller source term in transport equation (12).

It is noteworthy that the relative insensitivity of the simulation results on the detail of the inlet boundary conditions makes the test case under study appealing to modelers, because one (sometimes important) source of uncertainty is eliminated.

\subsection{TRANSPORTED PDF RESULTS - INFLUENCE OF THE PILOT FLAME MODEL}

Figure 11 shows mean temperature contour plots for the different energy source terms (10) and (11). As already mentioned, these energy source terms are necessary in order to obtain ignition in the numerical simulations. Clearly, the global differences between the flame shapes are small. The maximum temperature is slightly higher when $Q_{2}$ is applied and the flame is slightly shorter.

However, looking into more detail in the region close to the burner, large differences are observed. Figure 12, showing the turbulent kinetic energy on the symmetry axis, indeed reveals a qualitatively completely different behavior. With source term $Q_{1}$, positioned in the region $10 \mathrm{~mm}<x<20 \mathrm{~mm}$, a steep increase of k is observed close to the burner, followed by a decrease and a second peak. With $Q_{2}$ on the other hand, a decrease of $k$ is observed first, followed by a less steep increase and then a decrease again (with only a small secondary peak). The reason for this completely different behavior is to be found in the region behind the rim between the central fuel jet and the primary air annulus. In the PPDF simulations, this region is at high temperature, due to the chemical equilibrium chemistry model. In the TPDF simulations, no reactions occur, unless an energy source term is supplied. With $Q_{1}$, this is not the case, close to the rim. As a consequence, the density is much higher than with $Q_{2}$ (where the energy is supplied in the mentioned region). This higher mean density leads to a much higher value of eddy viscosity and to much larger production of turbulent kinetic energy. This explains the first peak, which is absent with $Q_{2}$. The large level of $k$ goes hand in hand with large turbulent shear stresses. In the axial momentum equation, the most important term is the radial derivative of 

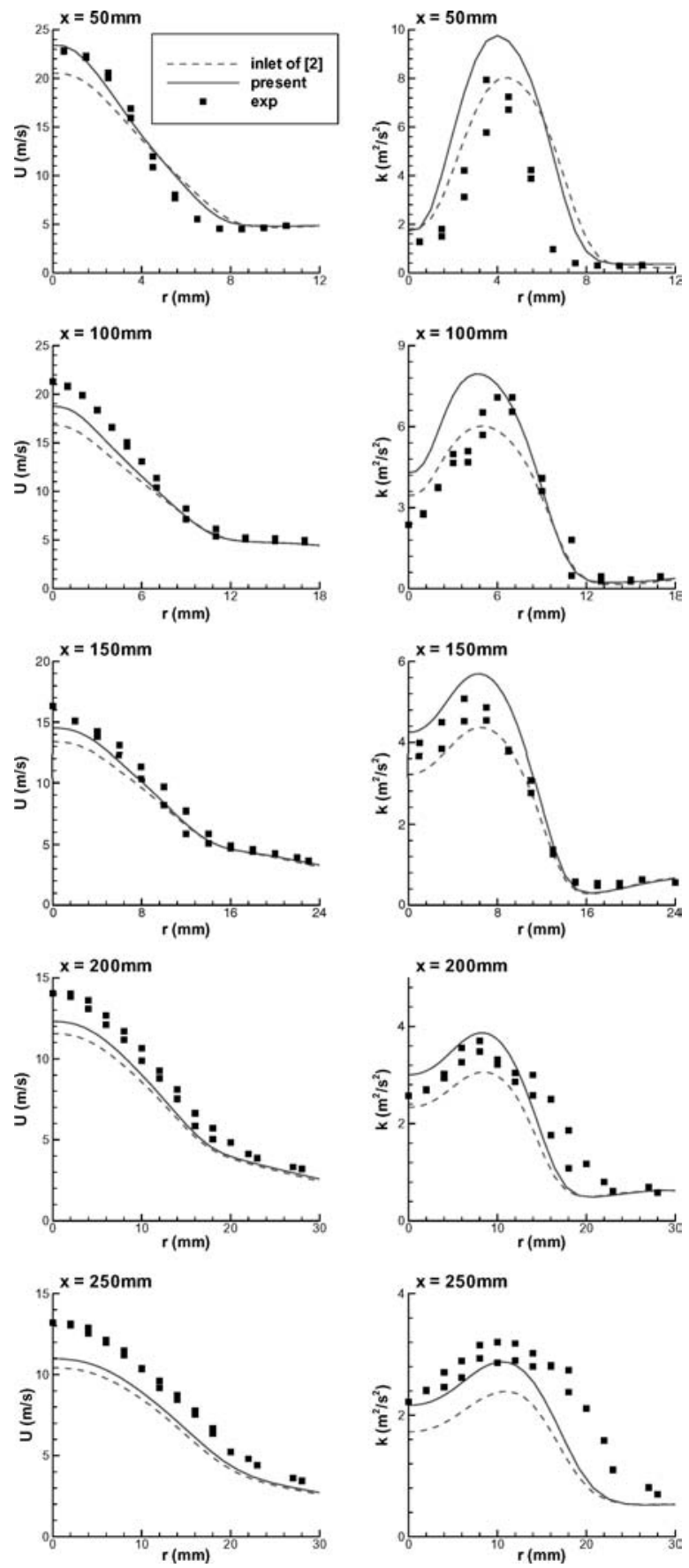

Figure 9. Radial profiles of mean axial velocity and turbulent kinetic energy: influence of inlet boundary conditions. 

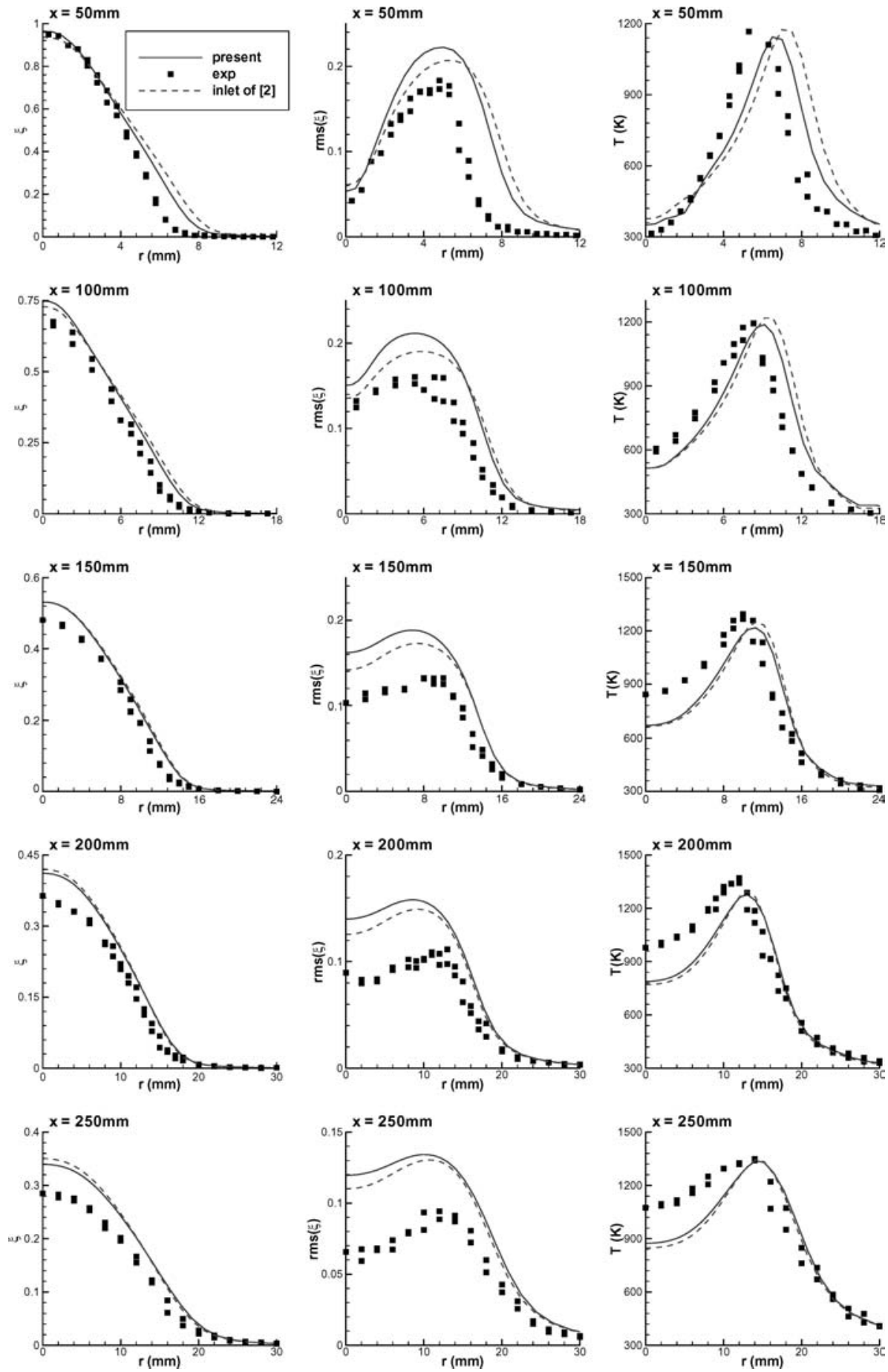

Figure 10. Radial profiles of mean mixture fraction, rms value of mixture fraction fluctuations and mean temperature: influence of inlet boundary conditions. 

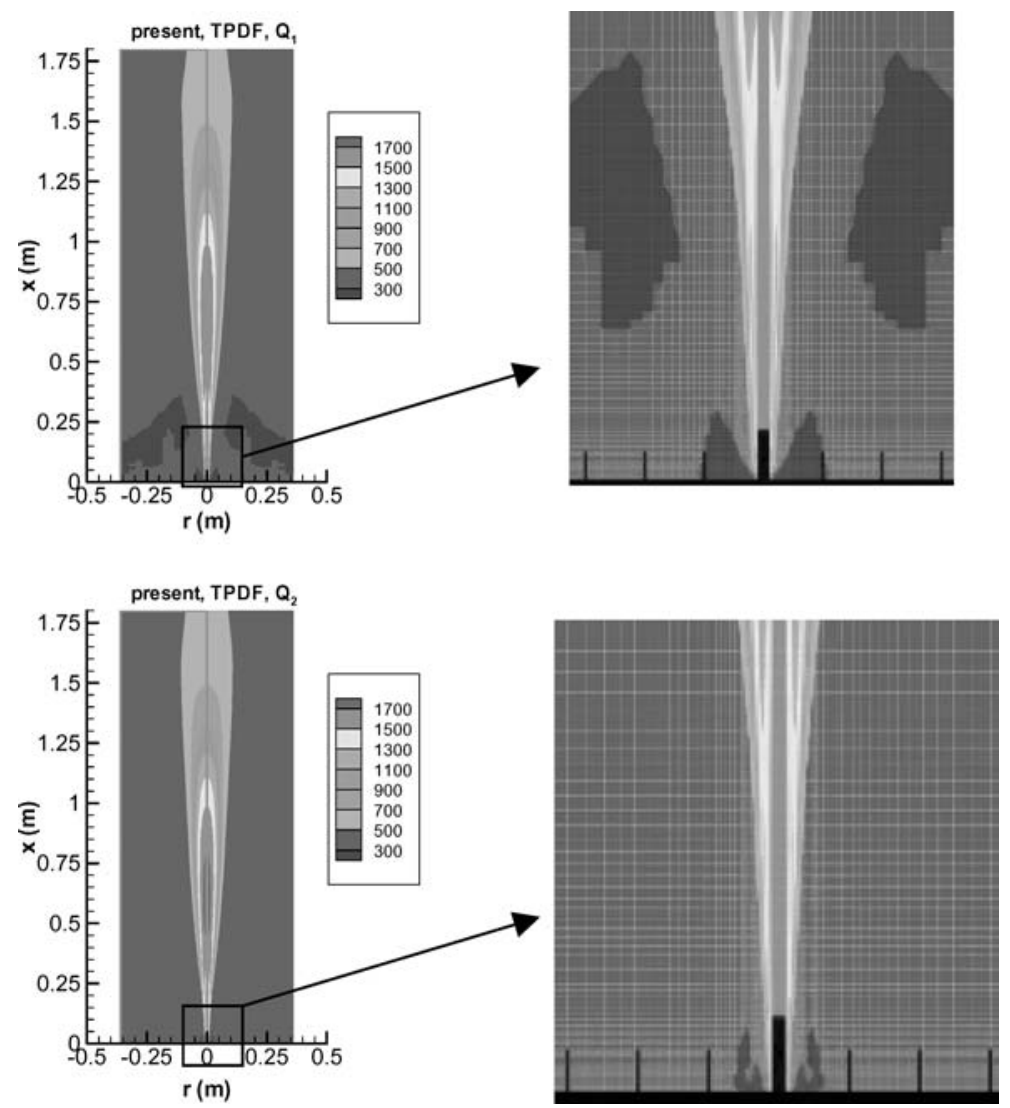

Figure 11. Contours of mean temperature: influence of pilot flame region on global quality of TPDF results.

the turbulent shear stress on the symmetry axis. This is the main force that decelerates the central jet. Clearly, the very large peak with $Q_{1}$ (Figure 12, right), severely slows down the central jet. This is indeed observed in Figure $13(x=50 \mathrm{~mm}$ and $x=100 \mathrm{~mm})$.

In this figure, it is illustrated that, at $x=50 \mathrm{~mm}$, the turbulent kinetic energy predicted by TPDF is much too high with $Q_{1}$ at the axis, while the shape with $Q_{2}$ is much better. The mean velocity profiles with $Q_{2}$ are in good agreement with the experimental data, while there is a serious under-prediction near the axis with $Q_{1}$ at $x=50 \mathrm{~mm}$ and $x=100 \mathrm{~mm}$, due to the too strong central jet deceleration. Further downstream, this under-prediction disappears, but for a wrong reason: the level of turbulent kinetic energy is under-estimated (due to too small mean velocity gradients), so that there is too little diffusion. Indeed, the spreading rate is too low at $x=200 \mathrm{~mm}$ and $x=250 \mathrm{~mm}$.

The above is confirmed in Figure 14, showing the mean mixture fraction and mean temperature. At $x=50 \mathrm{~mm}$, the mean mixture fraction predicted by TPDF 

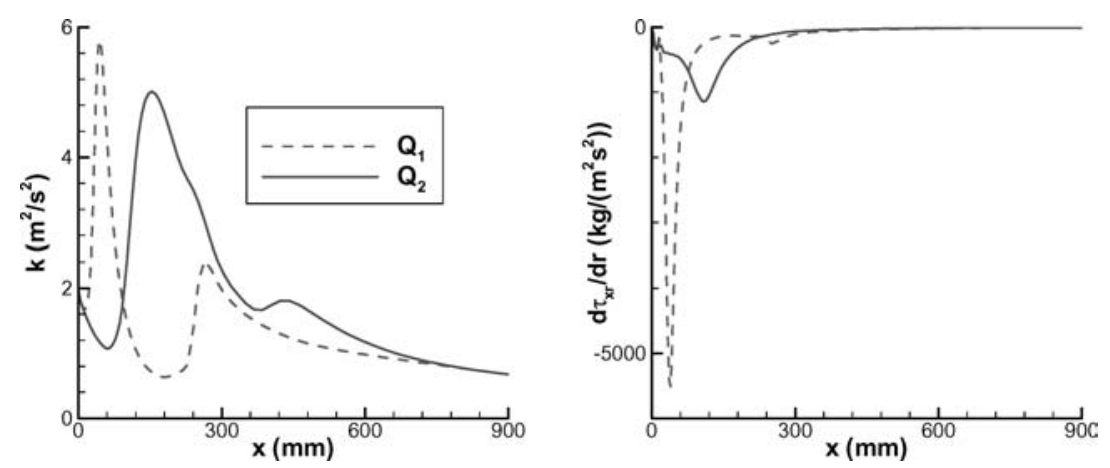

Figure 12. Axial profiles of turbulent kinetic energy and radial derivative of the turbulent shear stress on the axis: influence of pilot flame region.

is strongly under-predicted with $Q_{1}$ near the axis, due to the under-estimation of the mean axial velocity and due to excessive diffusion. Because the density in the region near the axis is too high (mean temperature under-predicted near the axis), while it is too low away from the axis (mean temperature over-predicted for $r>6 \mathrm{~mm}$ ), the momentum of the central jet is relatively too strong. The central jet is not sufficiently decelerated as was already observed in Figure 13 (over-prediction of mean axial velocity at the downstream positions). Here, the effect is stronger due to the combination of over-estimated density (under-estimated mean temperature): the product $\rho U$ is strongly over-estimated, so that there is far too much convection in Equation (3), leading to excessive values for mean mixture fraction near the axis at the downstream positions.

With $Q_{2}$, the reasoning is slightly more subtle. Here, the momentum of the central jet is well reproduced near the burner, due to the good agreement for the mean axial velocity and the mean temperature. However, the temperature outside this central region is over-predicted, so that the mean density is under-estimated and again the shear stresses, slowing down the central jet, are not strong enough. Consequently, an over-estimation of the mean mixture fraction near the axis at $x=200 \mathrm{~mm}$ and $x=250 \mathrm{~mm}$ is observed with $Q_{2}$, too, be it much less pronounced than with $Q_{1}$. Finally, the peak temperature radial position illustrates that the spreading rate with $Q_{2}$ is good, while it is under-estimated with $Q_{1}$ at $x=200 \mathrm{~mm}$ and $x=250 \mathrm{~mm}$.

Figure 15 shows profiles for the rms value of the mixture fraction fluctuations at $x=100 \mathrm{~mm}$ and $x=150 \mathrm{~mm}$. The mixture fraction variance has been determined in each computational cell by taking the sum of the squares of the differences between the individual particle mixture fraction value and the cell mean mixture fraction value, divided by the number of particles in the cell. With $Q_{2}$, a global over-prediction is observed, due to the too high level of turbulent kinetic energy and the too steep gradients of mean mixture fraction. Both aspects cause excessive transport of particles in physical space due to turbulent diffusion (Equation (6)) and raise the variance. With $Q_{1}$, this is not observed, due to the large under-prediction 
B. MERCI ET AL.
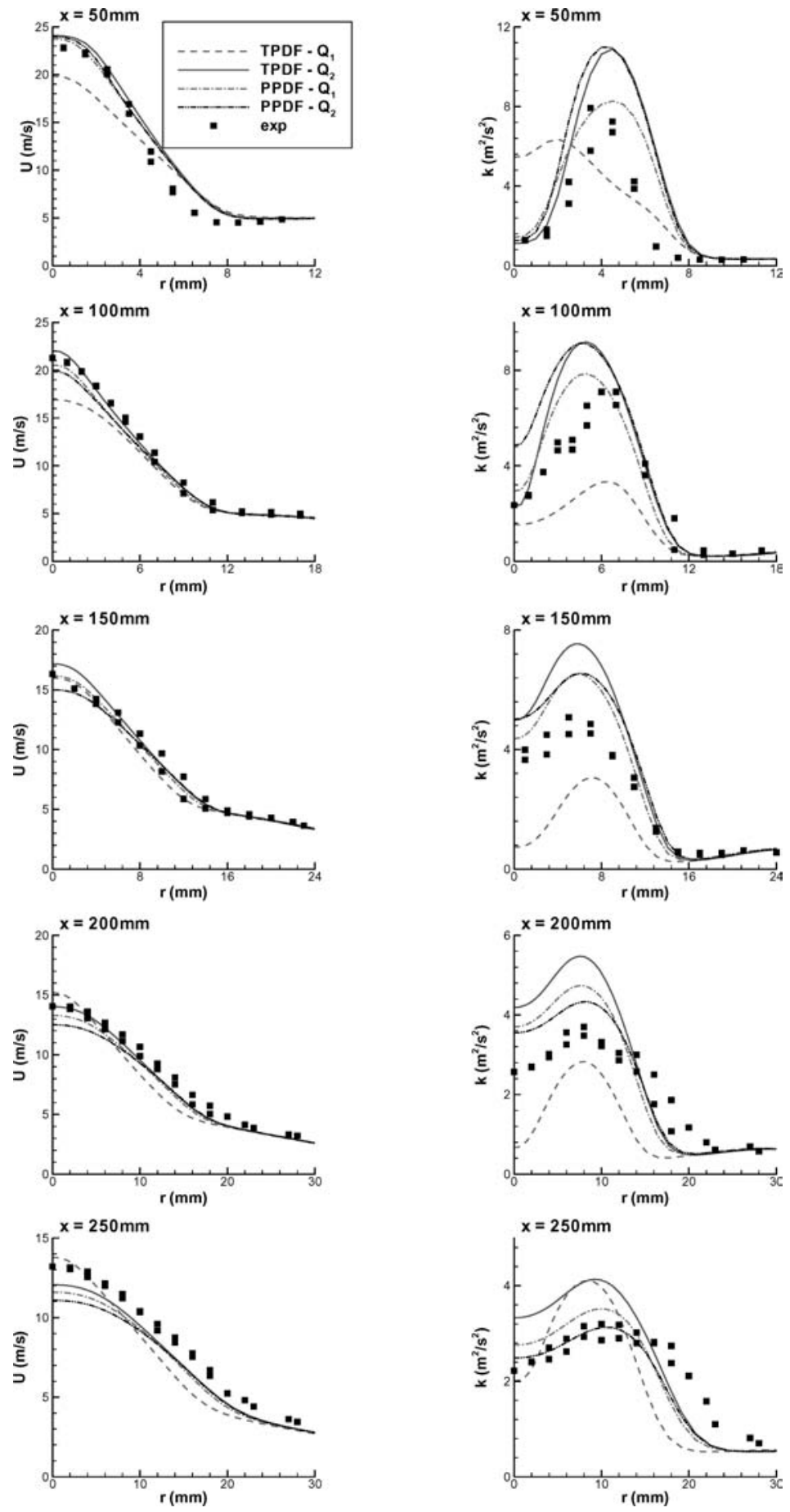

Figure 13. Radial profiles of mean axial velocity and turbulent kinetic energy: influence of pilot flame region. 

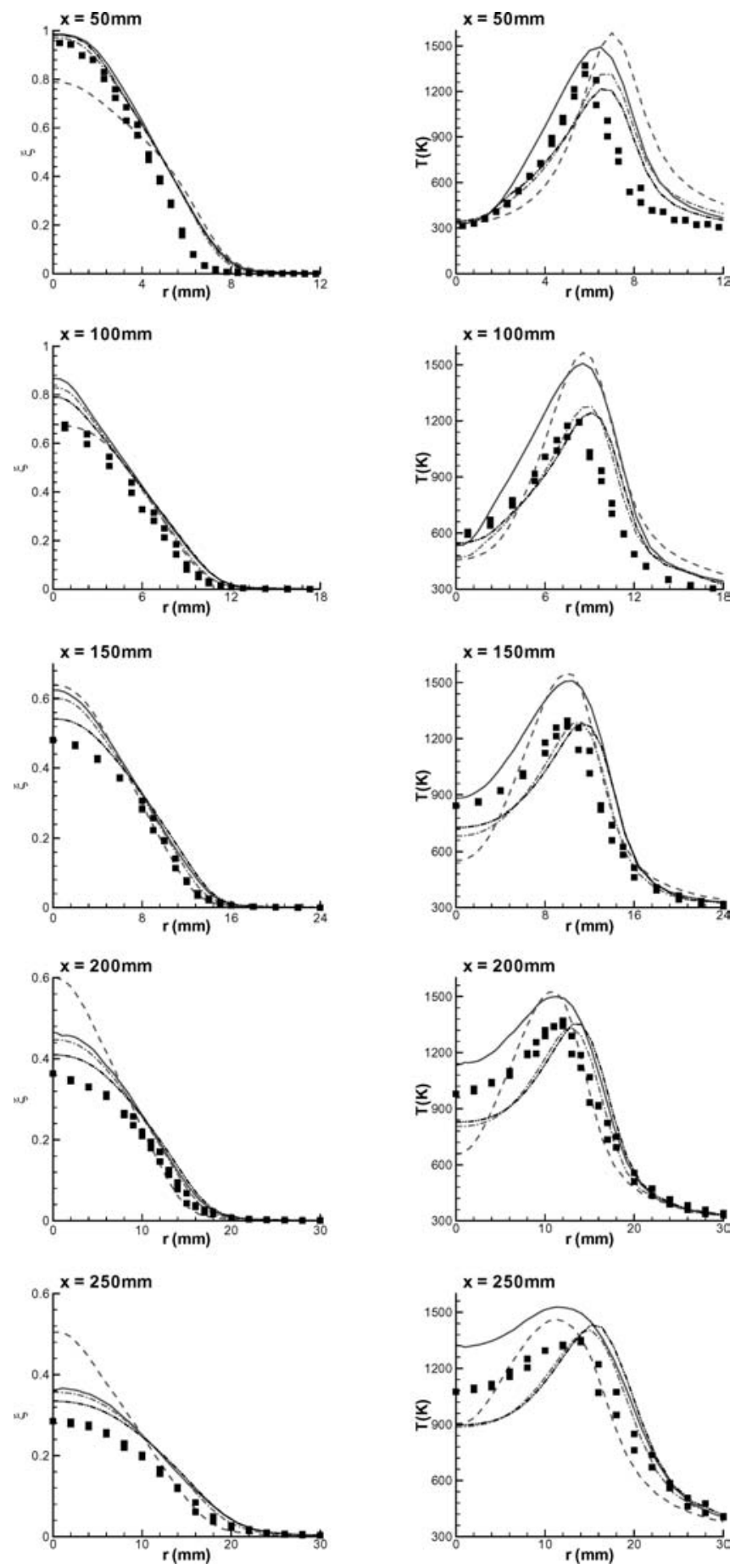

Figure 14. Radial profiles of mean mixture fraction variance and mean temperature: influence of pilot flame region. (Legend as in Figruer 13). 

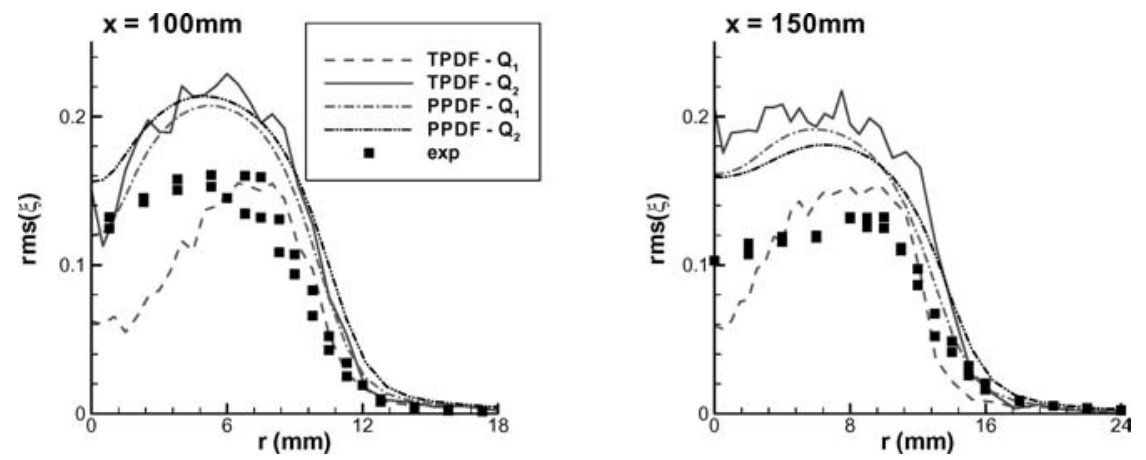

Figure 15. Radial profiles of rms value of mixture fraction fluctuations: influence of pilot flame region.

of turbulent kinetic energy. This also explains the incorrect dip of mixture fraction variance near the axis. It is interesting to make the link with transport Equation (4) for the mixture fraction variance. The turbulent diffusion transport term in physical space implies the production term in Equation (4), proportional to the square of the gradient of mean mixture fraction and to the turbulent diffusivity. Indeed, large values for any of these quantities lead to higher values of mixture fraction variance. The scalar dissipation rate term in Equation (4) corresponds to the micro-mixing term in Equation (6) and reduces local mixture fraction variance.

In Figures 13-15, comparisons are also made with PPDF results with the two heat source terms. The first observation is that the differences between the sets of PPDF results are much smaller than for the TPDF results. The differences with the profiles of Figures 4 and 5 are also relatively small, in particular for the mean temperature. This is to be expected: the thermal power of the heat sources is only $1 \%$ of the total flame thermal power. As explained above, the differences between the TPDF results are much larger, due to the large influence on the flow and mixing field.

Let us now focus on the profiles with source term $Q_{2}$. The mean axial velocity component is larger in the TPDF simulations and consequently in better agreement with the experimental data. The main reason is the over-prediction of mean temperature for $r>4 \mathrm{~mm}$, so that the central jet is less strongly decelerated than in the PPDF simulations. A phenomenon, counter-acting the above, is the overprediction of temperature (i.e., lower density and less momentum) near the axis for $x>150 \mathrm{~mm}$ in the TPDF results, in contrast to the PPDF results. Globally, both TPDF and PPDF are in good agreement with the experiments.

The higher mean axial velocity level and corresponding larger radial gradients hereof, are reflected in higher levels of turbulent kinetic energy with the TPDF method. Again, the shapes of the profiles are good for both PPDF and TPDF.

The differences between the mean mixture fraction profiles are explained by means of the mean axial velocity component again, in the context of mean convection-diffusion equation (3). The PPDF results are better. Here, we restrict ourselves to the use of standard model constant values. 

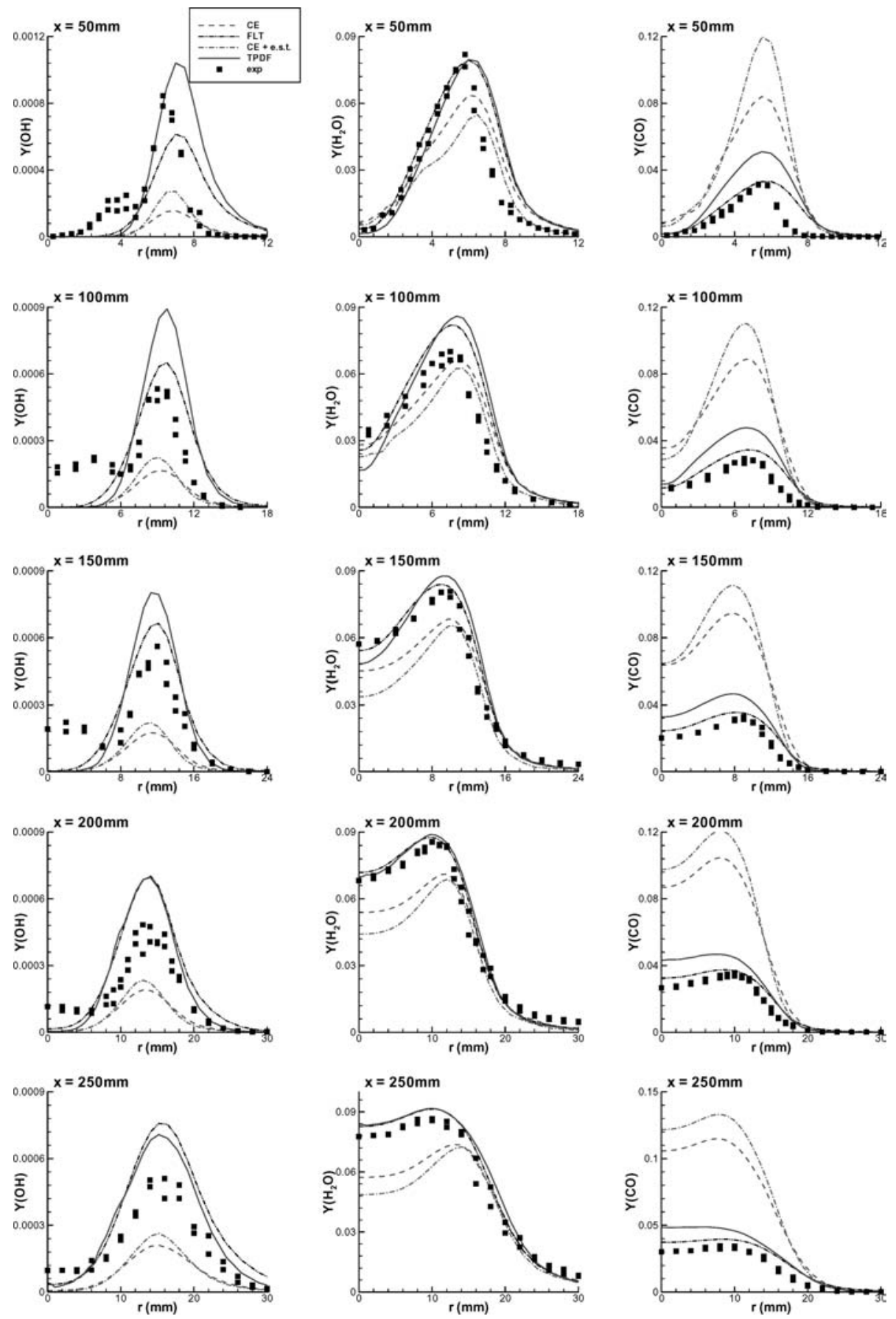

Figure 16. Radial profiles of mean mass fractions $\mathrm{CO}_{2}, \mathrm{H}_{2} \mathrm{O}$ and $\mathrm{CO}$ : comparison between PPDF and TPDF results. (CE: chemical equilibrium; FLT: laminar flamelet model; e.s.t.: energy source term $Q_{2}$ ). 
For TPDF, we observe global over-prediction of mean temperature at the rich side of stoichiometric conditions. The reasons are twofold: on the one hand, the $\mathrm{C}_{1}$ skeletal chemistry model [16] leads to excessive temperatures; on the other hand, the micro-mixing model, with $C_{\phi}=2$, under-predicts conditional fluctuations [18], so that the mean temperature is too high. Still, the results are comparable to what was obtained in [2]. With PPDF on the other hand, under-prediction is observed on the rich side, which is due to the applied chemical equilibrium chemistry model. All these findings are completely in line with the discussion of Figure 7. This is not surprising, since the laminar flamelet model was based on the $\mathrm{C}_{1}$ skeletal scheme.

The profiles of the rms value of the mixture fraction fluctuations (Figure 15) are very similar. This is logical, since in the scalar TPDF model, the turbulent viscosity is used in the random walk of the particles and as such determines the local variance of the mixture fraction. Since the eddy viscosity is similar in the PPDF and TPDF simulations, the profiles must be very similar, so that Figure 15 can also be regarded as some kind of consistency test.

Finally, Figure 16 shows profiles for different species. Obviously, there are large differences when different chemistry models are used. Agreement with the experimental data is much better when the $\mathrm{C}_{1}$ skeletal scheme is used (or the laminar flamelet model that is based on it). Addition of the heat source term influences the species, but the effect is by far not as strong as impact of the choice of the chemistry model. Note that the major species $\mathrm{H}_{2} \mathrm{O}$ is very well reproduced, while $\mathrm{CO}$ and $\mathrm{OH}$ are over-estimated. These are well-known deficiencies of the $\mathrm{C}_{1}$ skeletal scheme. The very strong over-prediction of CO in the PPDF results is consistent with the under-prediction of mean temperature: combustion is insufficiently complete, with too high levels of $\mathrm{CO}$ and too low levels of $\mathrm{CO}_{2}$ (not shown).

Obviously, there is still room for improvement in the chemistry model in the TPDF simulations. Our aim is to apply the Augmented Reduced Mechanism (ARM) of [27], in order to reduce shortcomings from the chemistry model. The simulations have been performed with the commercial CFD package FLUENT and first attempts with ARM were not successful. The $\mathrm{C}_{1}$ skeletal scheme on the contrary was easy to use. This is still a subject of investigation. Another aspect is the micro-mixing term. A comprehensive study, comparing the IEM model, the modified Curl's model and the EMST model, has been performed and the results are discussed in [18]. The value of the model constant $\mathrm{C}_{\phi}$ is discussed in that paper, too. In the current paper, we did not go into these issues, because we feel that the reasoning with respect to the topics that are dealt with remains consistent for the chemistry and micro-mixing models as described above.

\section{Conclusions}

An extensive set of simulation results has been presented for the piloted jet diffusion flame 'Delft Flame III'. Both the pre-assumed and the transported scalar PDF method have been applied and compared to each other. 
The global quality of the applied turbulence model, in terms of mean turbulent flow and mixing field predictions, was illustrated for both cold and reacting flows. Apart from an over-prediction of mixture fraction variance, as well as a slight overprediction of the spreading rate of the mean mixture fraction, the agreement with the experimental data is good with the present model.

Comparison of results obtained with chemical equilibrium to steady laminar flamelet modeling reveals that the differences in the flow and mixing fields are much smaller than observed with different turbulence models.

A procedure has been described for the rigorous construction of appropriate inlet boundary conditions for the specific test case under study. However, their detail is not important and their influence is much smaller than the choice of the turbulence model. This feature makes the test case particularly interesting for numerical simulations.

When the transported PDF approach is used to model the turbulence-chemistry interaction, the flame numerically extinguishes when the thermal power of the pilot flames is not supplied. A model, as a volumetric energy source term in the mean enthalpy transport equation, has been presented. It was illustrated that the influence on the mean flow and mixing field is large close to the burner, through the effect on the turbulent shear stress. On the global flame shape, the influence is relatively small.

\section{Appendix: Turbulence Model Description}

For many flow types and aspects in practice, a first order, but non-linear, expression for the turbulent stresses in terms of the local mean strain rate and vorticity tensors (5), is sufficient to obtain accurate results for a wide variety of flow types:

$$
-\bar{\rho} \widetilde{v}_{\mathrm{i}}^{\pi} v_{\mathrm{j}}^{\prime \prime}=2 \mu_{\mathrm{t}} S_{\mathrm{ij}}-\frac{2}{3} \rho k \delta_{\mathrm{ij}},
$$

with the eddy viscosity:

$$
\mu_{\mathrm{t}}=\rho\left(c_{\mu} f_{\mu}+\frac{c_{1}}{4} \tau_{\mathrm{t}}^{2}\left(\Omega^{2}-S^{2}\right)\right) k \tau_{t} .
$$

In (2), $f_{\mu}$ is a damping function, forcing the eddy viscosity to zero at solid boundaries (low-Reynolds model formulation). The turbulent time scale is defined as:

$$
\tau_{t}=\frac{k}{\varepsilon}+\sqrt{\frac{\mu}{\rho \varepsilon}},
$$

where the last term is added to avoid singularities near the solid boundaries (lowReynolds formulation).

The non-linearity in (A.1) is visible in the second term of (A.2), which accounts for the effect of rotation or streamline curvature on turbulence. Depending on the convex or concave nature of the streamline curvature, turbulence production is 
reduced or augmented. This is captured by the second term in (A.2), with the expression for $c_{1}$ :

$$
\left\{\begin{array}{l}
S \geq \Omega: c_{1}=-\min \left(40 c_{\mu}^{4} ; 0.15\right) \\
S<\Omega: c_{1}=-\min \left(\min \left(40 c_{\mu}^{4} ; 0.15\right) ; \frac{4 f_{\mu} c_{\mu}}{\left(\Omega^{2}-S^{2}\right) \tau_{t}^{2}}\right)
\end{array}\right.
$$

Calibration of the factor 40 and limiting the value to 0.15 has been done on a rotating fully developed channel. The last limit in the second line of (A.4) is introduced in order to prevent the turbulent viscosity (A.2) from becoming negative. In the test case under study, streamline curvature is not strong and the second term in (A.2) is small, compared to the first term, so that it can actually be neglected here.

The non-linearity in (A.1) is also hidden in the expression for $c_{\mu}$, which is taken from [11]:

$$
c_{\mu}=\left(4+A_{\mathrm{s}} \eta\right)^{-1},
$$

with:

$$
\begin{aligned}
S_{\mathrm{ij}} & =\frac{1}{2}\left(\frac{\partial v_{\mathrm{i}}}{\partial x_{\mathrm{j}}}+\frac{\partial v_{\mathrm{j}}}{\partial x_{\mathrm{i}}}\right)-\frac{1}{3} \frac{\partial v_{\mathrm{m}}}{\partial x_{\mathrm{m}}} \delta_{\mathrm{ij}} ; \quad \Omega_{\mathrm{ij}}=\frac{1}{2}\left(\frac{\partial v_{\mathrm{i}}}{\partial x_{\mathrm{j}}}-\frac{\partial v_{\mathrm{j}}}{\partial x_{\mathrm{i}}}\right) \\
S & =\left(2 S_{\mathrm{ij}} S_{\mathrm{ij}}\right)^{1 / 2} ; \quad \Omega=\left(2 \Omega_{\mathrm{ij}} \Omega_{\mathrm{ij}}\right)^{1 / 2} ; \quad \eta=\tau_{\mathrm{t}}\left(S^{2}+\Omega^{2}\right)^{1 / 2} \\
A_{\mathrm{s}} & =\sqrt{3} \cos \phi ; \quad \phi=\frac{1}{3} \arccos (\sqrt{6} W) ; \quad W=\frac{2^{3 / 2} S_{\mathrm{ij}} S_{\mathrm{jk}} S_{\mathrm{ki}}}{S^{3}}
\end{aligned}
$$

More important for the test case under study are the (steady-state) transport equations for the turbulence quantities, particularly for the turbulent dissipation rate:

$$
\left\{\begin{aligned}
\frac{\partial}{\partial x_{\mathrm{m}}}\left(\rho k v_{\mathrm{m}}\right)= & P_{\mathrm{k}}-\rho \varepsilon+\frac{\partial}{\partial x_{\mathrm{m}}}\left[\left(\mu+\frac{\mu_{\mathrm{t}}}{\sigma_{\mathrm{k}}}\right) \frac{\partial k}{\partial x_{\mathrm{m}}}\right] \\
\frac{\partial}{\partial x_{\mathrm{m}}}\left(\rho \varepsilon v_{\mathrm{m}}\right)= & \left(1-f_{R_{\mathrm{y}}}\right) c_{\varepsilon 1} \frac{P_{\mathrm{k}}}{\tau_{\mathrm{t}}}+f_{R_{\mathrm{y}}} C_{1} \rho S \varepsilon-c_{\varepsilon 2} f_{2} \rho \frac{\varepsilon}{\tau_{\mathrm{t}}} \\
& +\frac{\partial}{\partial x_{\mathrm{m}}}\left[\left(\mu+\frac{\mu_{\mathrm{t}}}{\sigma_{\varepsilon}}\right) \frac{\partial \varepsilon}{\partial x_{\mathrm{m}}}\right]+E
\end{aligned}\right.
$$

The transport equation for $k$ is standard. The model parameters are given in Table II. They are all standard or retrieved from [11], except for $c_{\varepsilon 2}$. This parameter contains a rotation term that makes sure that the $\varepsilon$ transport equation remains consistent for high rotation rates. For the test case under study, this is completely irrelevant.

Noteworthy is the source term in the $\varepsilon$ transport equation, which is a blending of the 'traditional' source term (i.e. the turbulent kinetic energy production term, divided by the turbulence time scale) in the neighborhood of solid boundaries and a source term, derived from a modeled enstrophy transport equation [11]. This 
second source term part ensures that the well-known plane jet - round jet anomaly disappears $[10,11]$, while the first part avoids strong dependence on inlet boundary conditions and irregular behavior near solid boundaries. The exact shape of the blending function $f_{R y}$ is not crucial. Here, it is based on the normalized distance $R_{y}=\rho \sqrt{k} y / \mu$ from the nearest solid boundary: it goes from zero to one as $R_{y}$ goes from 1000 to 2000 .

The low Reynolds source term $E$ is derived from the standard $k-\omega$ model, as described in $[6,10]$ :

$$
E=-1.8\left(1-f_{\mu}\right)\left(\mu+\frac{\mu_{\mathrm{t}}}{\sigma_{\varepsilon}}\right) \frac{\partial k}{\partial x_{\mathrm{m}}} \frac{\partial \tau_{\mathrm{t}}^{-1}}{\partial x_{\mathrm{m}}} .
$$

This term improves near-wall behaviour in general but has little effect for the test case under study, since it is only non-zero near the nozzle exit due to the factor $\left(1-f_{\mu}\right)$.

The boundary conditions at a solid boundary are $k=0$ and $\varepsilon=2 \frac{\mu}{\rho}(\partial \sqrt{k} / \partial n)^{2}$. Table II provides an overview of the different model constants.

\section{Acknowledgments}

The first author is Postdoctoral Researcher of the Fund of Scientific Research Flanders (Belgium) (FWO-Vlaanderen). Part of the work was financed by the Fund of Scientific Research - Flanders (Belgium) (FWO-Vlaanderen) through FWOproject G.0070.03.

\section{References}

1. Peeters, T.W.J., Stroomer, P.P.J., de Vries, J.E., Roekaerts, D.J.E.M. and Hoogendoorn, C.J., Proc. Combust. Inst. 25 (1994) 1241-1248.

2. Nooren, P.A., Wouters, H.A., Peeters, T.W.J., Roekaerts, D., Maas, U. and Schmidt, D., Combust. Theory Modelling 1 (1997) 79-96.

3. Nooren, P.A., Versluis, M., van der Meer, T.H. and Barlow, R.S., Appl. Phys. B 71 (2000) 95-111.

4. van Veen, E.H. and Roekaerts, D., Combust. Sci. Technol. 175 (2003) 1893-1914.

5. http://www.ca.sandia.gov/TNF.

6. 2nd Workshop on Aerodynamics of Steady-State Combustion Chambers and Furnaces (A.S.C.F.), Pisa, November 28-29, 1996. Final Report. Edited by G. Mariotti and S. Tiribuzi, ENEL, Direzione Studi E Richerche, Centro Ricerca Termica.

7. Oksanen, A., Turbulence-combustion interaction in two different gas-fired furnaces. In: Proceedings of the 4th European Conference on Industrial Furnaces and Boilers, Espinho, Porto, Portugal, April 1-4, 1997.

8. Mantzaras, J. and van der Meer, T.H., Combust. Flame 110(1-2) (1997) 39-53.

9. Merci, B., Vierendeels, J., Dick, E., Roekaerts, D. and Peeters, T.W.J., Combust. Flame 126(1-2) (2001) 1533-1556.

10. Merci, B. and Dick, E., Flow Turb. Combust. 68(4) (2002) 335-358.

11. Merci, B., Dick, E. and De Langhe, C., Combust. Flame 131(4) (2002) 465-468.

12. Merci, B. and Dick, E., Int. J. Heat Mass Transfer 46(3) (2003) 469-480. 
13. Merci, B., De Langhe, C., Lodefier, K. and Dick, E., J. Thermophys. Heat Transfer 18(1) (2004) 100-107.

14. Shih, T.H., Liou, W.W., Shabbir, A., Yang, Z. and Zhu, J. Comput. Fluids 24(3) (1995) 227238.

15. Pope, S.B., Prog. En. Combust. Sci. 11 (1985) 119-192.

16. Correa, S.M., Combust. Flame 93(1-2) (1993) 41-60.

17. Subramaniam, S. and Pope, S.B., Combust. Flame 115 (1998) 487-514.

18. Merci, B., Roekaerts, D., Naud, B. and Beishuizen, N., "Study of the Performance of Different Micro-Mixing Models in Transported Scalar PDF Simulations of a Piloted Jet Diffusion Flame", Comb. Flame (in review).

19. Muradoglu, M. and Pope, S.B., AIAA J. 40(9) (2002) 1755-1763.

20. Dopazo, C., Phys. Fluids 18 (1975) 397-404.

21. Janicka, J., Kolbe, W. and Kollman, W., J. Non-Equil. Thermodyn. 4 (1979) 47-66.

22. Ren, Z. and Pope, S.B., Combust. Flame 136(1-2) (2004) 208-216.

23. Stroomer, P.P.J., Turbulence and $\mathrm{OH}$ structures in flames, PhD Thesis, Delft University of Technology (1995).

24. Jones, W.P. and Launder, B.E., Int. J. Heat Mass Transfer 15(2) (1972) 301-314.

25. Launder, B.E., Morse, A., Rodi, W. and Spalding, D.B., Technical Report NASA SP-311 (1972).

26. Bilger, R.W., Combust. Flame 80 (1990) 135-149.

27. Sung, C.J., Law, C.K. and Chen, J.-Y., Proc. Combust. Inst. 27 (1998) 295-304. 